\title{
Novel redispersible nanosuspensions stabilized by co-processed nanocrystalline cellulose-sodium carboxymethyl starch for enhancing dissolution and oral bioavailability of baicalin
}

This article was published in the following Dove Press journal:

International Journal of Nanomedicine

\section{Jin $\mathrm{Xie}^{1, *}$ \\ Yijing Luo ${ }^{1, *}$ \\ Yang Liu' \\ Yueqin $\mathrm{Ma}^{1,2}$ \\ Pengfei Yue ${ }^{1, *}$ \\ Ming Yang'}

'Key Laboratory of Modern Preparation of TCM, Ministry of Education, Jiangxi University of

Traditional Chinese Medicine,

Nanchang 330004, China;

${ }^{2}$ Department of Pharmaceutics, 94th

Hospital of People's Liberation Army,

Nanchang, China

*These authors contributed equally to this work
Correspondence: Pengfei Yue Jiangxi University of Traditional Chinese Medicine, 1688 Meilingdadao Road,

Nanchang 330004, China

Emailypfpharm@I26.com
Background: To improve the dissolution and bioavailability of poorly soluble drugs, novel nanosuspensions using co-processed nanocrystalline cellulose-sodium carboxymethyl starch (NCCS) as a synergetic stabilizer were first designed.

Methods: Co-processed NCCS was prepared by means of homogenization. Poorly soluble baicalin (BCA) was used as a model drug. BCA nanosuspension (BCA-NS/NCCS) using co-processed NCCS as a dispersant was prepared via homogenization and further converted into the dried BCA nanosuspension particle (BCA-NP/NCCS) via spray drying. The influence of NCCS on the dispersion efficiency of BCA-NS/NCCS was investigated. Morphology and crystal characteristic of NCCS and BCA-NP were analyzed. The dissolution and bioavailability evaluation were performed to investigate the feasibility of NCCS as a stabilizer for BCA-NS/ NCCS and BCA-NP.

Results: The optimum 50\% concentration of NCCS (nanocrystalline cellulose [NCC]:sodium carboxymethyl starch $[S C S]=60: 40$ ) could be mostly beneficial for formation and stability of BCA-NS/NCCS. NCCS could completely prevent aggregation of BCA-NP during spray drying and enhance the redispersibility as well as dissolution of spray-dried BCA-NP, which might be attributed to "brick-concrete"-based barrier effect of NCCS and the swelling capacity of superdisintegrant SCS. The crystal state of NCC and BCA presented in BCA-NP/NCCS remained unchanged during the homogenization. The BCA-NP/NCCS exhibited a fast dissolution rate and significantly enhanced bioavailability of BCA. The $\mathrm{AUC}_{(0-\infty)}$ of the BCA-NP/ $\operatorname{NCCS}(8,773.38 \pm 718.18 \mu \mathrm{g} / \mathrm{L} \cdot \mathrm{h})$ was 2.01 times $(P<0.05)$ as high as that of the crude BCA $(4,354.61 \pm 451.28 \mu \mathrm{g} / \mathrm{L} \cdot \mathrm{h})$.

Conclusion: This study demonstrated that novel surfactant-free nanosuspensions could be prepared using co-processed NCCS as a synergetic stabilizer and also provided a feasible strategy to improve the dissolution and oral bioavailability of poorly soluble drug.

Keywords: nanocrystalline cellulose, Pickering nanosuspensions, nanocrystals, solid particles stabilizer, oral bioavailability

\section{Introduction}

Nanosuspensions or nanocrystal suspensions (NSs) are considered one of the most valuable strategies for formulation of poorly soluble drugs. NS has numerous advantages such as increasing the solubility and dissolution velocity of poorly soluble drug owing to its small particle size less than $1 \mu \mathrm{m}$ in diameter and a large specific surface area. ${ }^{1-3}$ NS is a carrier-free colloidal suspension that contains nanosized drug particles 
and minimal water-soluble stabilizers, typically produced in the forms of suspensions. ${ }^{4}$ Water-soluble stabilizers such as polymers and/or surfactants are generally used to prevent aggregation of drug nanocrystals during homogenization and thus ensure physical stability of the drug suspensions at storage. ${ }^{5}$ Hydroxypropyl methylcellulose, hydroxypropyl cellulose, and polyvinylpyrrolidone are the most conventionally used soluble and nonionic polymers, which can stabilize drug nanocrystals via steric barrier effect, whereas anionic surfactants can prevent from aggregation of nanocrystals dependent on increasing the electrostatic repulsion among nanocrystals. ${ }^{6}$ However, the physical stability issues of drug NS such as particle size growth and aggregation upon prolonged storage increasingly restricted its application. ${ }^{7,8}$ One effective strategy to circumvent such difficulty is to transform liquid nanosuspensions into dried nanosuspensions (also named as solid nanocrystals) by means of spray drying or freeze-drying., ${ }^{9,10}$ The main challenge is that the dried nanosuspension particles or solid nanocrystals are able to reform immediately the original NS after rehydration and improve the dissolution of poorly soluble drug. ${ }^{11,12}$

An ideal stabilizer/dispersant system is not only required to ensure proper physical stability of drug NS at the suspension state and enhance the redispersibility of dried nanosuspension particles after drying but also exhibit excellent safety and compatibility. ${ }^{13,14}$ For example, ionic surfactants in oral formulations present challenges such as incompatibilities with other ionic molecules, sensitivity to $\mathrm{pH}$, salt or temperature changes, gastrointestinal tract irritation, and toxicity. ${ }^{15-17}$ Furthermore, in the absence of surfactants and their synergistic action with the polymers, excessively high polymer concentration may be required to stabilize drug nanosuspensions, which could negatively affect the redispersion and dissolution of drug. ${ }^{18,19}$ Therefore, development of novel "green" stabilizer/dispersants that help to impart physical stability, good redispersibility, and safety to dried drug nanosuspension particles is paid more and more attention.

Nanocrystalline cellulose (NCC; Figure 1A) is regarded as an ideal biomaterial due to its unique properties, such as uniform rod-like shape, high surface area, liquid crystalline behavior, biocompatibility, biodegradability, sustainability, and nontoxic carbohydrate-based nature. ${ }^{20} \mathrm{NCC}$ has especially attracted increasingly attention in the past decades as drug delivery systems. ${ }^{21}$ For example, NCC is used as a solid particle stabilizer of oil-in-water Pickering emulsions. ${ }^{22,23}$ NCC-based hydrogels could control the behavior of prolonged drug release, which was attributed to the "obstruction effect". ${ }^{24,25}$ Furthermore, co-processed modification and combination of NCC particles with water-soluble cellulose derivatives can be very necessary to allow for successful production of colloidal formulation. ${ }^{26,27} \mathrm{Hu}$ et al ${ }^{28}$ demonstrated that Pickering emulsions stabilized by NCC preadsorbed with water-soluble methylcellulose derivatives could be converted into the oil powder that easily redispersed into the emulsion oil droplets in water. The novel aspect of the current work is to first use co-processed NCCS as "synergetic stabilizer" dispersant for production of drug NS. Sodium carboxymethyl starch (SCS; Figure 1B) is widely used for fast disintegration and dissolution of poorly soluble drug nanocrystals, which is a crosslinked semisynthetic polymer with high swelling capacities. ${ }^{29-31}$ Therefore, co-processed nanocrystalline cellulose and sodium carboxymethyl starch (NCCS) as a synergetic stabilizer may be an interesting strategy for design of drug nanosuspensions. The ideal NCCS is suitable for drug nanosuspensions according to the following requirements: 1) excellent viscosity and suspension ability (suitable for homogenization of nanocrystals); 2) shortterm stability (10 days) after homogenization (allowing the
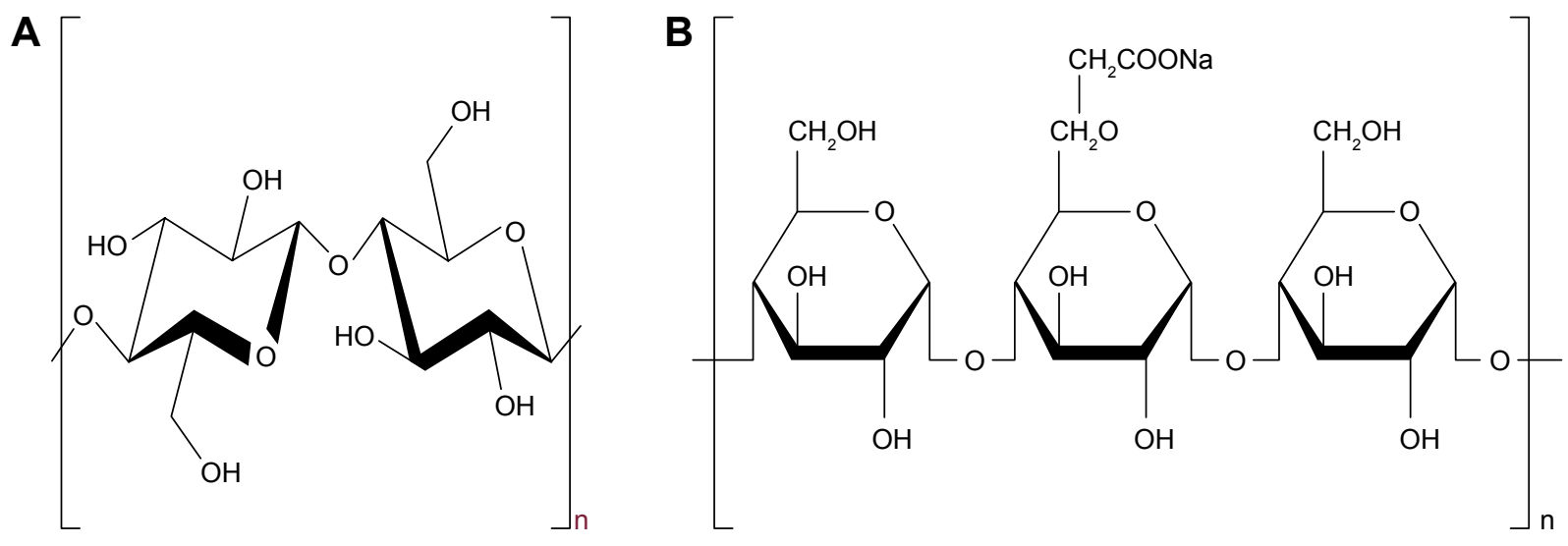

Figure I Chemical structure of NCC (A) and SCS (B).

Abbreviations: NCC, nanocrystalline cellulose; SCS, sodium carboxymethyl starch. 

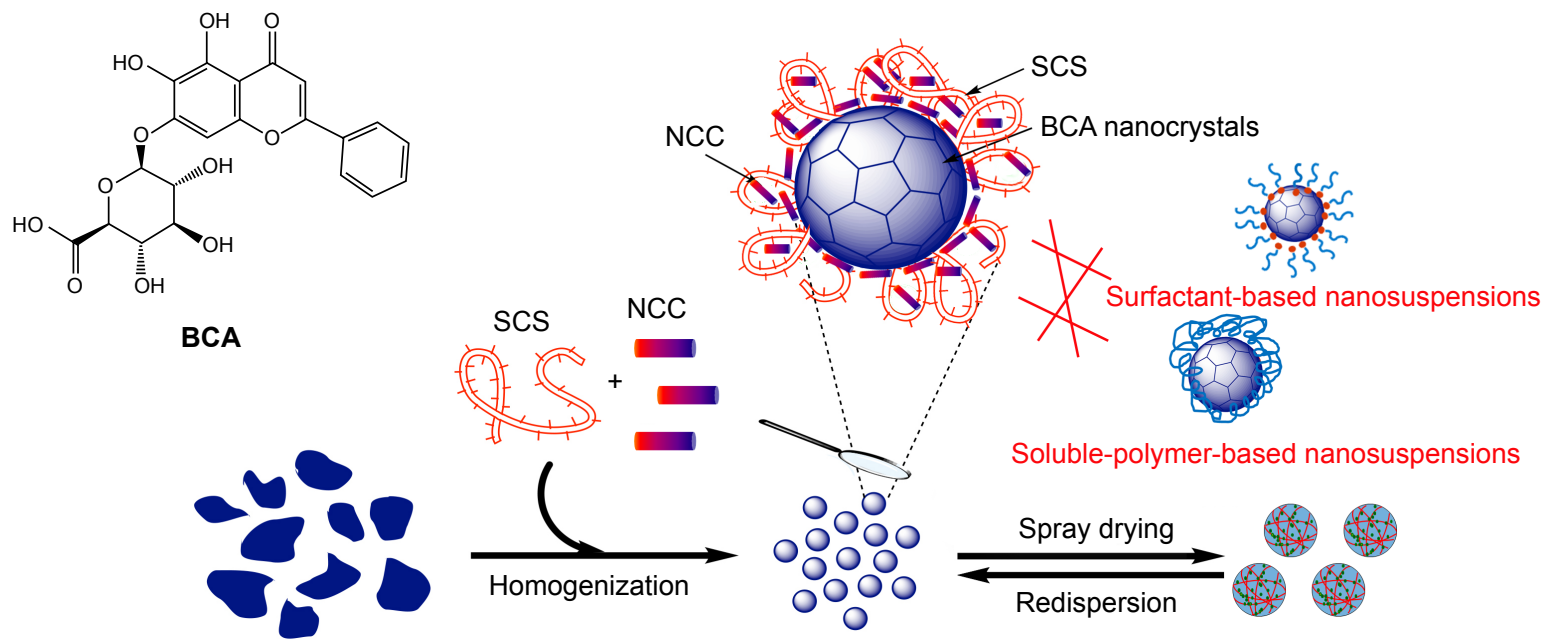

BCA

BCA-NS/NCCS

BCA-NP/NCCS

Figure 2 The schematic image of BCA-NS stabilized by NCCS.

Abbreviations: BCA, baicalin; BCA-NS, BCA nanosuspension; NCCS, nanocrystalline cellulose-sodium carboxymethyl starch; SCS, sodium carboxymethyl starch; NCC, nanocrystalline cellulose; BCA-NP, BCA nanosuspension particle.

follow-up spray-drying process); and 3) excellent reconstitution properties (ie, conservation of particle size and dissolution after reconstitution).

Baicalin (BCA; Figure 2) is a naturally occurring flavonoid. It was isolated from the roots of Scutellaria baicalensis Georgi, which has long been used in Chinese medicine. BCA has shown strong physiological activities, including antioxidant and anti-inflammatory properties, as well as antimicrobial and antifungal activities. ${ }^{32-34}$ Moreover, $\mathrm{BCA}$ is restricted in formulations owing to its poor water solubility $(<0.05 \mathrm{mg} / \mathrm{mL})$ and oral bioavailability $(<10 \%) .{ }^{35}$ To overcome these issues of BCA, several nano-based formulation approaches have been developed, such as solid nanocrystals, ${ }^{36}$ solid self-microemulsifying delivery system, ${ }^{37}$ nanoemulsions, and solid lipid nanoparticles. ${ }^{38,39}$ Although these formulation technologies have greatly improved bioavailability of BCA, there are some safety issues associated with the use of surfactants or polymers. ${ }^{40}$

In our current study, as illustrated in Figure 2, BCA was used as a model drug, the novel BCA nanosuspension (BCA-NS) stabilized by NCCS was first designed, in order to enhance the drug dissolution, redispersibility, and bioavailability of BCA. The main objectives of this article were to prepare BCA-NS stabilized by NCCS (BCA-NS/ NCCS) and further convert it into dried BCA nanosuspension particle (BCA-NP/NCCS) via spray drying. Co-processed NCCS, NCC, or SCS was, respectively, used as a stabilizer, and their performance was compared for preparation of BCA-NP. We also aimed to evaluate the morphology of the BCA-NP/NCCS visualized by transmitting electron microscopy (TEM) and scanning electron microscopy (SEM), characterize the solid state of BCA-NP by X-ray diffraction (XRD) and differential scanning calorimetry (DSC), and further investigate the dissolution and oral bioavailability of BCA-NP.

\section{Materials and methods Materials}

BCA (purity $>98 \%$ ) was purchased from Chengdu Herbpurify Co., Ltd. (Chengdu, China). Microcrystalline cellulose (MCC) used for the preparation of NCC was commercially obtained from Fengli Jingqiu Pharmaceutical Co., Ltd. (Beijing, China). Crosslinked SCS (DST ${ }^{\circledR}$ Type A) was purchased from Chineway Pharmaceutical Co., Ltd (Hefei, China).

\section{Co-processed NCCS suspensions}

According to the formula (S1-1-S8-1) listed in Table 1, MCC and SCS were dispersed in the water at 1,000 rpm for 30 minutes. The gained coarse suspension of MCC and SCS was homogenized at a high pressure using a piston-gap high pressure homogenizer (AH-1000D; ATS Engineering Inc., Seeker, Canada). First, 30 cycles at 200 bar and 20 cycles at 400 bar were conducted as pre-milling step, and afterward, 30 cycles at 800 bar were run to obtain the NCCS colloidal suspensions.

\section{Production of BCA-NS/NCCS and BCA-NP/NCCS}

According to the formula (S1-2-S8-2) listed in Table 1, BCA coarse powder $1 \%(\mathrm{w} / \mathrm{v})$ was dispersed in the prepared 
Table I Formula design of NCCS and BCA-NS/NCCS

\begin{tabular}{|c|c|c|c|c|}
\hline \multirow[t]{2}{*}{ Sample } & \multirow{2}{*}{$\begin{array}{l}\text { Concentrations } \\
\text { of dispersant } \\
(\%, w / v)\end{array}$} & \multicolumn{2}{|c|}{$\begin{array}{l}\text { Ratios of MCC and } \\
\text { SCS in dispersant }\end{array}$} & \multirow[t]{2}{*}{$\begin{array}{l}\text { BCA } \\
(\%, w / v)\end{array}$} \\
\hline & & MCC (\%) & SCS (\%) & \\
\hline SI-I & 0.25 & 100 & 0 & 0 \\
\hline SI-2 & & & & 1 \\
\hline S2-I & & 80 & 20 & 0 \\
\hline S2-2 & & & & I \\
\hline S3-I & & 60 & 40 & 0 \\
\hline S3-2 & & & & I \\
\hline S4-I & & 40 & 60 & 0 \\
\hline S4-2 & & & & I \\
\hline S5-I & & 20 & 80 & 0 \\
\hline S5-2 & & & & I \\
\hline S6-I & 0.5 & 60 & 40 & 0 \\
\hline$S 6-2$ & & & & I \\
\hline S6-3 & & 0 & 100 & I \\
\hline S7-I & 1 & 60 & 40 & 0 \\
\hline S7-2 & & & & 1 \\
\hline S8-I & 2 & 60 & 40 & 0 \\
\hline S8-2 & & & & 1 \\
\hline
\end{tabular}

Abbreviations: BCA, baicalin; NCCS, nanocrystalline cellulose-sodium carboxymethyl starch; BCA-NS, BCA nanosuspension; MCC, microcrystalline cellulose; SCS, sodium carboxymethyl starch.

NCCS with a series of ratio of MCC and SCS. The obtained mixture was first disintegrated into coarse BCA suspension by a high shear homogenizer (FLUKO ${ }^{\circledR}$ FA25; Essen, Germany) at 16,000 rpm for 5 minutes. Then, the BCA coarse suspension was homogenized at a high pressure using a piston-gap high pressure homogenizer (AH-1000D; ATS Engineering Inc., Seeker, Canada) with a continuous water cooling system (ATS Engineering Limited, Suzhou, China) that was used to maintain the temperature of the suspension at the room temperature. First, 30 cycles at 200 bar and 30 cycles at 500 bar were conducted as a pre-milling step, and afterward, 30 cycles at 1,000 bar were applied to obtain the BCA-NS/NCCS. For comparison with BCA-NS/NCCS (S6-2), BCA-NS with $0.5 \%$ SCS (S6-3) was prepared as follows: BCA coarse powder $1 \%(\mathrm{w} / \mathrm{v})$ was dispersed in $0.2 \%$ SCS suspensions and further homogenized into BCA-NS as described above, and then, remaining $0.3 \%$ SCS was added to the homogenized BCA-NS/SCS suspension.

The BCA-NP/NCCS was prepared by spraying the BCA-NS/NCCS through the nozzle of a Buchi mini spray dryer (model B290; Buchi Laboratoriums-Technik AG, Flawil, Switzerland). The process parameters were set as follows: the nozzle cap orifice diameter was $0.7 \mathrm{~mm}$, inlet temperature was set as $120^{\circ} \mathrm{C}$, the feed rate was $2 \mathrm{~mL} \cdot \mathrm{min}^{-1}$, and the atomizing air flow was $5 \mathrm{~m}^{3} \cdot \mathrm{h}^{-1}$. The BCA-NP/ NCCS particles were separated from the drying air in the cyclone $\left(60^{\circ} \mathrm{C}\right.$ outlet temperature) and deposited at the bottom of the collector. They were collected and kept at room temperature for future testing and evaluation. Furthermore, the dried NCCS also was obtained for follow-up characterization of its crystals state by means of process parameters as described above.

\section{Particle size and zeta potential measurements of NCCS and BCA-NS/NCCS}

The determination of particles size was performed on a Mastersizer Microplus (Malvern Instruments, Malvern, $\mathrm{UK})$, which has a working range of $0.050-550 \mu \mathrm{m}$. Analysis of the diffraction patterns was done using the Mie model ("standard" presentation: dispersant refractive index $=1.33$, real particle refractive index $=1.5295$, imaginary particle refractive index=0.1). The particle sizes $D_{10}, D_{50}$, and $D_{90}$ were determined in order to access the variation in the size of particles. $D_{10}, D_{50}$, and $D_{90}$ are the volumetric diameters where the $10 \%, 50 \%$, and $90 \%$ of the population lies below each value, respectively. All measurements were performed in triplicate.

The zeta potential of NCCS and BCA-NS/NCCS was determined by the dynamic light scattering (Nano ZS; Malvern Instruments). The samples were first diluted to a concentration of $\sim 0.05 \% \mathrm{w} / \mathrm{v}$ using deionized water in order to avoid multiple scattering effects. All measurements were performed in triplicate. The zeta potential was reported as the average and SD of measurements.

\section{Rheological evaluation of NCCS}

The rheological properties of the different NCCS dispersions (Table 1) were assessed using the AR2000ex rheometer (TA Instruments, Crawley, UK). Samples were placed between the gap of the cone and plate, and the gap was closed gradually. This unit gives either a controlled shear rate or a controlled shear stress in a cone and plate rheometry format. A $60 \mathrm{~mm}$ cone ( $1^{\circ}$ angle geometry) was used for all of the measurements and to provide uniform shearing to the samples. Samples were transferred to the plate with the minimum of shearing. The equilibration time before measurement was 2 minutes. The elastic module $\left(\mathrm{G}^{\prime}\right)$ and viscous module $\left(\mathrm{G}^{\prime \prime}\right)$ were then determined. All the measurements were performed at $37^{\circ} \mathrm{C} \pm 0.1^{\circ} \mathrm{C}$.

\section{The suspension stability of BCA-NS/NCCS}

To ensure the stabilization of BCA-NS/NCCS at the suspension state for allowing follow-up spray drying, the shortterm stability was evaluated in terms of the redispersibility 
index (RDI) of BCA-NS after storage. BCA-NS/NCCS was placed in tightly sealed screw vials immediately at $25^{\circ} \mathrm{C}$ for 10 days. Then, the samples were redispersed by handshaking (10 seconds) and withdrawn for determination of particles' size as described in the "Production of BCA-NS/NCCS and BCA-NP/NCCS" section.

\section{The redispersibility of BCA-NP/NCCS}

A total of $100 \mathrm{mg}$ of BCA-NP/NCCS powders were redispersed in a $40 \mathrm{~mL}$ vial with $20 \mathrm{~mL}$ pure water, and then, the suspension was shaken for 5 minutes at 250 strokes/ minute (horizontal spectrophoshaker, Agitelec SL200). Samples were withdrawn for determination of particles' size as described in the "Production of BCA-NS/NCCS and BCA-NP/NCCS" section. The RDI of BCA-NP/NCCS was calculated by the following equation: ${ }^{24}$

$$
\mathrm{RDI}=\frac{D}{D_{0}}
$$

where $D_{0}$ is the volume-weighed $\left(D_{50}\right)$ mean particle size of the precursor BCA-NS/NCCS directly prior to spray drying and $D$ is the corresponding value of redispersed suspensions from spray-dried BCA-NP/NCCS. An RDI of $\sim 1$ indicates that BCA-NP/NCCS can completely recover back to the original BCA-NS/NCCS after rehydration.

\section{In vitro dissolution evaluation of BCA-NS/NCCS and BCA-NP/NCCS Drug determination of BCA-NS/NCCS and BCA-NP/NCCS}

The concentration of BCA in BCA-NS/NCCS and BCA-NP/ NCCS was assayed by HPLC according to the reported method. ${ }^{36}$ The chromatographic separation was achieved on Hypersil ODS-2 $(25 \times 4 \mathrm{~mm}, 5 \mu \mathrm{m})$. The chromatographic conditions was set as follows: the detection wavelength: $280 \mathrm{~nm}$; the mobile phase: acetonitrile $0.2 \%(\mathrm{v} / \mathrm{v})$ and aqueous phosphoric acid 47:53, v/v; the injection volume: $20 \mu \mathrm{L}$; the flow rate: $1 \mathrm{~mL} / \mathrm{min}$; and the detection temperature: $30^{\circ} \mathrm{C}$.

\section{In vitro dissolution of BCA-NS/NCCS and BCA-NP/NCCS}

The dissolution profiles of three batches of BCA, BCA-NS/ NCCS, and BCA-NP/NCCS containing the same amount of BCA (60 mg) were evaluated, respectively. According to the CP XC paddle method, a dissolution apparatus (RC-8; Tianjin Guoming Medicine and Equipment Co., Inc., Tianjin, China) was used. In all, $900 \mathrm{~mL}$ of water at $37^{\circ} \mathrm{C}$ was used as a dissolution medium. The rotation speed of the paddles was set at $100 \mathrm{rpm}$. At predetermined time intervals $(1,3,5,10$, $15,20,30,60$ minutes), $2 \mathrm{~mL}$ samples were withdrawn and filtered through a $0.22 \mu \mathrm{m}$ filter membrane immediately. Simultaneously, equal blank medium was compensated immediately after the withdrawal. The amount of dissolved BCA in the sample solution was assayed as the above described HPLC method.

The similarity factor $\left(f_{2}\right)$ was used to assess the differences on dissolution profiles of different BCA formulations. ${ }^{18} f_{2}$ was calculated by the following equation:

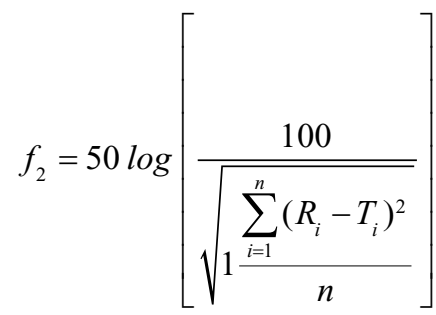

where $R_{i}$ represents the dissolution concentration of the compared formulation at predetermined time, $T_{i}$ represents the dissolution concentration of the developed formulation at the predetermined time, and $n$ represents the number of the dissolution time points. Generally, if $f_{2}$ value is much more than 50, the dissolution profile of the developed formulation is not significantly different from that of reference formulation.

\section{The morphology and solid state characterization of NCCS, BCA-NS/ NCCS, and BCA-NP/NCCS}

\section{TEM}

NCCS and BCA-NS/NCCS were analyzed by TEM (JEM1200EX; JEOL, Tokyo, Japan). The samples were diluted with a concentration of $\sim 0.05 \% \mathrm{w} / \mathrm{v}$ distilled water and placed on a copper grid. The grid was dried at room temperature and was evaluated with a electron microscope.

\section{SEM}

Morphology of the BCA-NP/NCCS was evaluated by an SEM (Hitachi X650; Hitachi Ltd., Tokyo, Japan). The samples were glued and solidified on metal sample plates by means of a carbon double-sided tape. All the samples were gold coated (thickness $\approx 15-20 \mathrm{~nm}$ ) by means of a sputter coater (Fison Instruments, Manchester, UK). The electrical potential was set as $2.0 \mathrm{kV}$ at $25 \mathrm{~mA}$ for 10 minutes. 
DSC

An analyzer DSC (Diamond DSC; PerkinElmer Inc., Waltham, MA, USA) was used to determine the peak melting temperature of the coarse BCA, dried NCCS, and BCA-NP/ NCCS. In all, 6-7 mg of powder samples was weighed before being placed in a sealed perforated aluminum pan and heated from $30^{\circ} \mathrm{C}$ to $260^{\circ} \mathrm{C}$ at a rate of $10^{\circ} \mathrm{C} / \mathrm{min}$, respectively. Nitrogen was used as the purge gas and protective gas at a flow rate of $50 \mathrm{~mL} / \mathrm{min}$ and $150 \mathrm{~mL} / \mathrm{min}$, respectively.

\section{Powder X-ray diffraction (PXRD)}

The crystallinity of the coarse BCA, dried NCCS particles, and BCA-NP/NCCS was analyzed using PXRD (PANalytical, Westborough, MA, USA). The samples were scanned for $2 \theta$ ranging from $5^{\circ}$ to $60^{\circ}$ at a scan rate of $0.2^{\circ} \mathrm{C} / \mathrm{min}$. Measurements were performed at a voltage of $40 \mathrm{kV}$ and $25 \mathrm{~mA}$.

\section{Stability of BCA-NP/NCCS}

The optimum BCA-NP/NCCS (S6-2) was placed in tightly sealed screw vials immediately, protected from light and placed at 4,25 , and $40^{\circ} \mathrm{C}$ for 6 months. The stability was evaluated in terms of the BCA content, redispersibility, and dissolution behavior of BCA-NP after storage. The BCA content in BCA-NP/NCCS was determined as described in "Drug determination of BCA-NS/NCCSand BCA-NP/ NCCS" section. The RDI and the similarity factor $\left(f_{2}\right)$ of dissolution curves of BCA-NP were evaluated as described in "The redispersiblity of BCA-NP/NCCS" section and "in vitro dissolution evaluation of BCA-NS/NCCS and BCA-NP/ NCCS" section, respectively.

\section{In vivo pharmacokinetic study of BCA-NS/NCCS and BCA-NP/NCCS}

The pharmacokinetic characterization of the optimum BCA-NP/NCCS (S6-2) was evaluated compared with crude BCA and the precursor BCA-NS/NCCS (S6-2). This study protocol was approved by the institutional ethics committee of Jiangxi University of Traditional Chinese Medicine. Animal welfare and experimental procedures were performed strictly in accordance with the Guide for the Care and Use of Laboratory Animals and the ethics regulations of Jiangxi University of Traditional Chinese Medicine.

The rats were housed at $25^{\circ} \mathrm{C} \pm 2{ }^{\circ} \mathrm{C}$, with $50 \% \pm 10 \%$ relative humidity and a 12-hour light-dark cycle. They were permitted free access to food and water until 12 hours prior to experiments. Male Wister rats with an average weight of $200 \pm 20 \mathrm{~g}$ were fasted 12 hours before administration.
A total of 18 rats were randomly divided into three groups of six animals each: coarse BCA suspension group, BCA-NS/NCCS group, and BCA-NP/NCCP group. The coarse BCA suspensions group was prepared by suspending the required amounts $(10 \mathrm{mg} / \mathrm{mL})$ of BCA in $0.5 \%$ Arabic gum aqueous solution. The redispersed BCA-NP/NCCS group was prepared by dispersing $1.5 \mathrm{~g}$ of BCA-NP/NCCS powder in $100 \mathrm{~mL}$ distilled water (equal to $10 \mathrm{mg} / \mathrm{mL}$ of BCA). BCA-NS/NCCS, BCA-NP/NCCS, and coarse BCA groups were orally administered at a dose of $100 \mathrm{mg} / \mathrm{kg}$.

At the predetermined intervals of $0.08,0.17,0.5,1,1.5$, $2,3,4,6,8,12$, and 24 hours, the blood sample of $0.25 \mathrm{~mL}$ was collected by retro orbital puncture. Plasma was obtained from whole blood in a heparinized tube via centrifugation at 4,000 rpm for 15 minutes and was frozen at $-20^{\circ} \mathrm{C}$ until analysis. The plasma concentrations of BCA were determined by a validated HPLC-MS/MS method. ${ }^{41}$ Carbamazepine was used as an internal standard (IS). The full scan spectra showed parent ion at $\mathrm{m} / \mathrm{z} 445 / 269$ for BCA and $\mathrm{m} / \mathrm{z}$ 237/194 for IS. An elution system of acetonitrile and $0.1 \%$ formic acid (45:55) was used at a flow rate of $0.2 \mathrm{~mL} \cdot \mathrm{min}^{-1}$.

The main pharmacokinetics parameters were calculated by DAS 2.0 software (Mathematical Pharmacology Professional Committee of China). ANOVA analysis was carried out using SPSS Statistics 21 software. Differences were considered as statistically significant if the $P$-value was $<0.05$.

\section{Results and discussion Influence of different concentrations of MCC and SCS on particle size of NCCS}

The particle sizes $\left(D_{10}, D_{50}\right.$, and $\left.D_{90}\right)$ of freshly prepared NCC (S1-1) are shown in Figure 3A. The results showed that the mean particle size $\left(D_{50}\right)$ of $\mathrm{NCC}(\mathrm{S} 1-1)$ in water was $846.13 \pm 0.16 \mathrm{~nm}$, which indicated that MCC could be disintegrated to nanosized particles via high-pressure homogenization. Production of NCC conventionally involves the acid-catalyzed hydrolysis of the cellulosic feedstock using concentrated, strong mineral acids. ${ }^{42}$ The cellulosic pulp or cotton linters can be usually used as raw materials for preparing NCC, but the properties of the NCC vary dependent on which acid is used for the hydrolysis. ${ }^{43} \mathrm{MCC}$ has also recently gained interest as a valid intermediate material for producing NCC materials. ${ }^{44}$ However, the $D_{50}$ of the redispersed NCC particles (S1-1) after storage was $1.213 \pm 0.16 \mu \mathrm{m}$. This meant that NCC particles easily formed the aggregation of large particles during storage.

To enhance stability of NCC during homogenization and storage, the water-soluble polymer SCS was introduced 

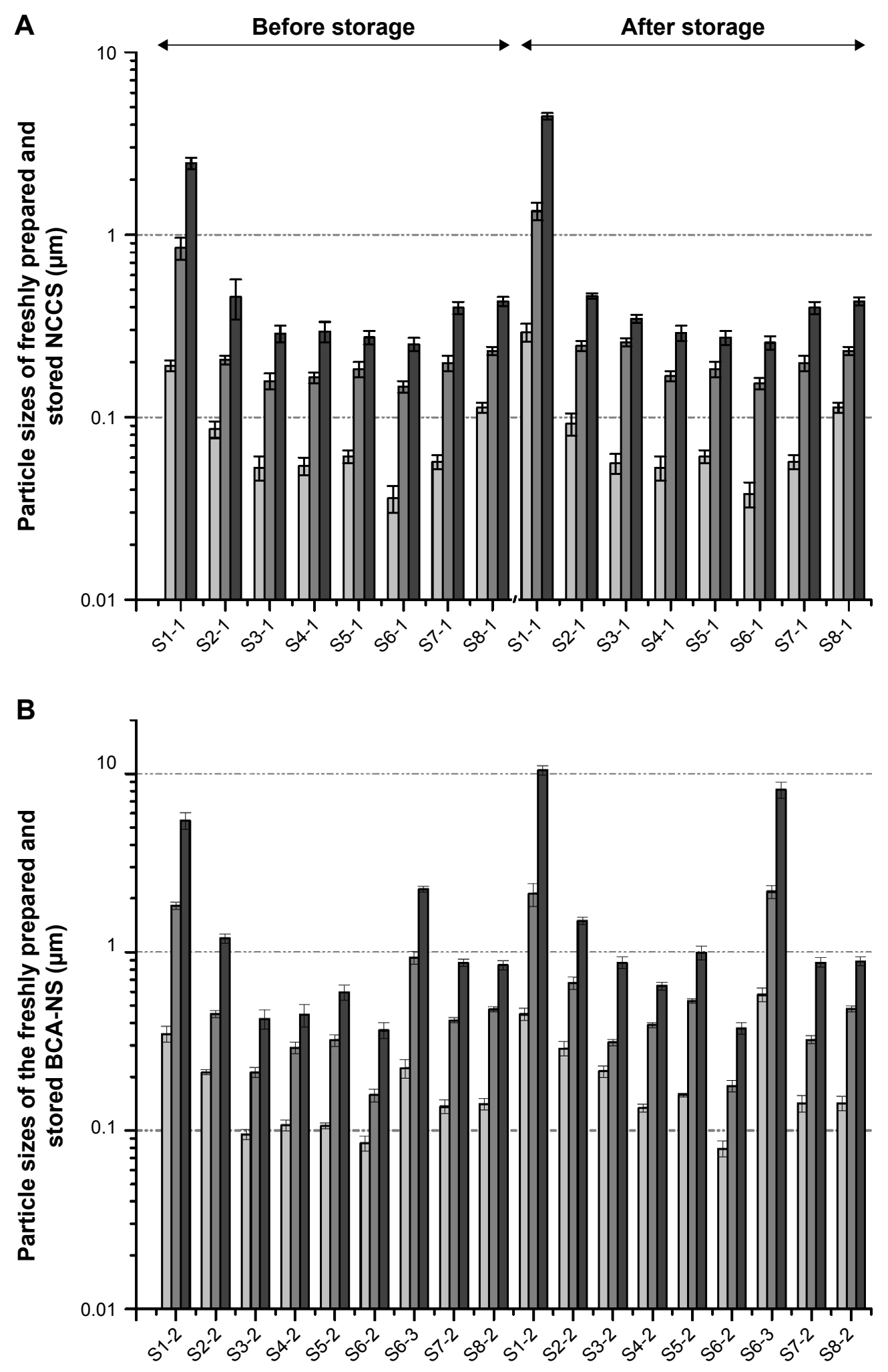

$$
\square D_{10} \square D_{50} \square D_{90}
$$

Figure 3 Particle sizes of NCCS (A) and BCA-NS/NCCS (B) before and after storage.

Note: $D_{10}, D_{50}$, and $D_{90}$ are the volumetric diameters where the $10 \%, 50 \%$, and $90 \%$ of the population lies below each value, respectively.

Abbreviations: BCA, baicalin; NCCS, nanocrystalline cellulose-sodium carboxymethyl starch; BCA-NS, BCA nanosuspension. 
into production of NCCS. The particle sizes $\left(D_{10}, D_{50}\right.$, and $D_{90}$ ) of NCCS (S2-1-S8-1) with different ratios of SCS are shown in Figure $3 \mathrm{~A}$. The mean particle size $\left(D_{50}\right)$ of coprocessed NCCS (S2-1-S8-1) with different ratios of MCC and SCS was $147.12 \pm 0.14 \mathrm{~nm}-231.12 \pm 0.11 \mathrm{~nm}$. The results showed that MCC could be disintegrated into much smaller NCC particles in the presence of SCS compared with those without SCS (S1). The particle sizes $\left(D_{10}, D_{50}\right.$, and $\left.D_{90}\right)$ of NCCS after short-term storage were not obviously increased compared with those of S1-1. These demonstrated that the presence of water-soluble polymer SCS could improve the particle-reduction efficiency of MCC during homogenization and enhance the suspension stability of NCCS. These were also thought to be related to the zeta potential of NCCS. The zeta potential of NCCS co-processed by different combinations of MCC and SCS was shown in Figure 4. The results showed that the zeta potential $(-45.8$ to $-85.7 \mathrm{mV})$ of different NCCS was significantly less than that of NCC $(-4.8$ $\mathrm{mV}$ ), which could be attributed to the presence of anionic polymer SCS. ${ }^{45}$ The zeta potential of NCCS was decreased with the increased concentration of NCCS.

However, with the increase in SCS ratio in dispersants (S4-1 and S5-1), the particle sizes $\left(D_{10}, D_{50}\right.$, and $\left.D_{90}\right)$ of NCCS were not significantly varied compared with those of NCCS (S3-1). The reason might be that the viscosity of
NCCS was increased along with the increase in SCS ratio, but which could decrease the efficiency of particle size reduction of NCC during homogenization. The particle sizes of the NCCS (S4-1 and S5-1) after short-term storage were not obviously increased compared with those of S3-1. Therefore, the moderate ratio of SCS (S3-1) might favor the formation of NCC during homogenization, which might become as strong as to make NCC stability, due to its steric effect and electrostatic repulsion effect. ${ }^{31}$

Furthermore, with the increase in stabilizer concentration, the particle sizes of NCCS for S7-1 and S8-1 were significantly increased compared with those of S6-1. However, the particle size of S6-1 was not significantly different from that of S3-1. These findings also demonstrated that the viscosity of suspensions was increased along with increase in NCCS concentration, but this could decrease the efficiency of particle size reduction of NCC during homogenization.

\section{Influence of different concentrations of NCCS on stability of BCA-NS/NCCS}

The particle sizes $\left(D_{10}, D_{50}\right.$, and $\left.D_{90}\right)$ of freshly prepared BCA-NS stabilized by different concentrations of NCCS are shown in Figure 3B. The results showed that the $D_{50}$ and $D_{90}$ of BCA suspensions (S1-2) using only NCC as a stabilizer were $1.823 \pm 0.142 \mu \mathrm{m}$ and $5.458 \pm 0.142 \mu \mathrm{m}$, respectively,

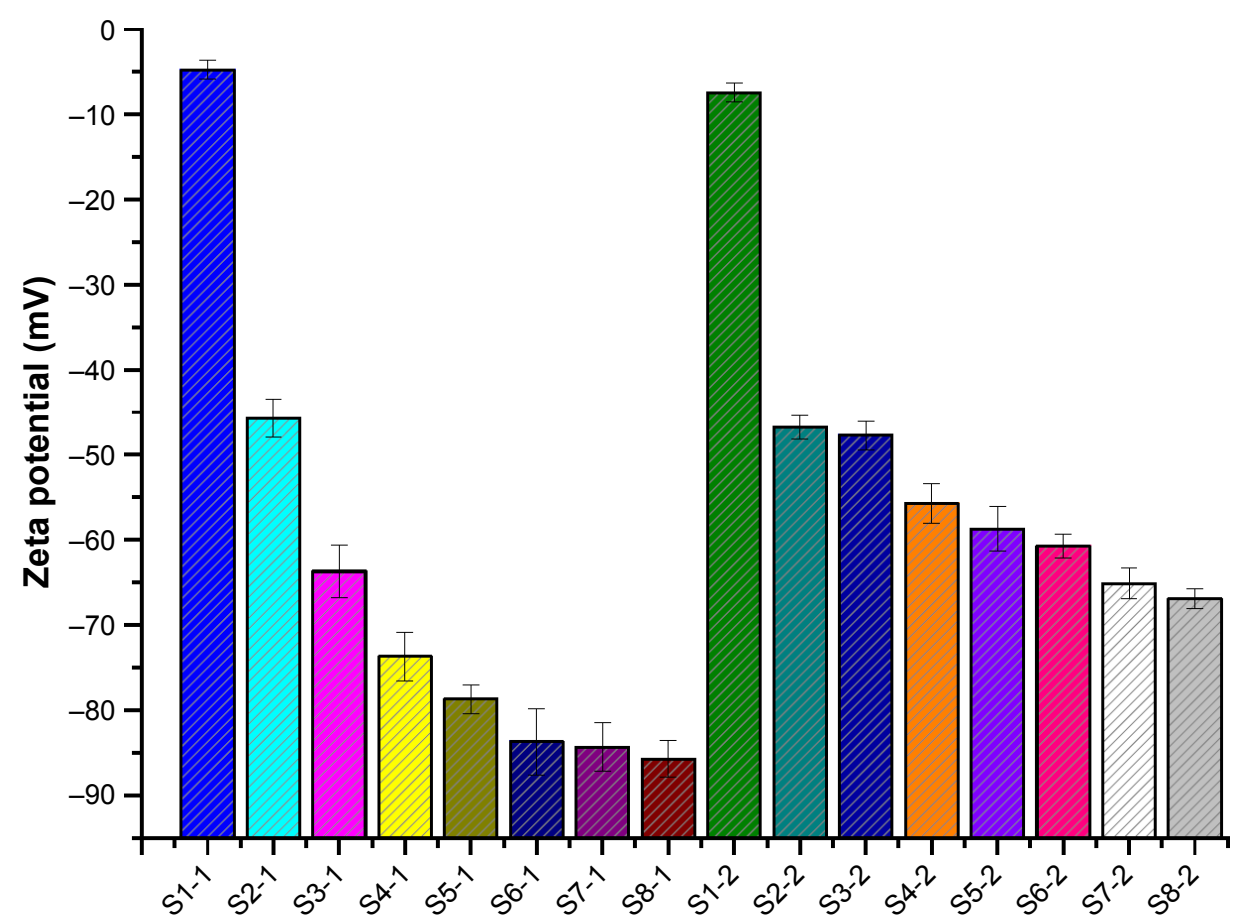

Figure 4 The zeta potential of the freshly prepared NCCS and BCA-NS/NCCS that was co-processed by different ratios of MCC and SCS. Abbreviations: BCA, baicalin; NCCS, nanocrystalline cellulose-sodium carboxymethyl starch; BCA-NS, BCA nanosuspension; MCC, microcrystalline cellulose; SCS, sodium carboxymethyl starch. 
which meant that BCA-NS could be not successfully prepared and only depended on NCC.

It could be also seen that the particle size $D_{50}$ of BCA-NS/ NCCS (S2-2, S3-2, S4-2, S5-2, S6-2, S7-2 and S8-2) in water was $212.21 \pm 0.14 \mathrm{~nm}-481 \pm 0.23 \mathrm{~nm}$. This demonstrated that BCA could be disintegrated to nanosized particles stabilized by NCCS. However, the particle size of BCA-NS/NCCS (S4-2 and S5-2) was not significantly varied compared with that of NCCS (S3-2). The reason might be that the increased viscosity of NCCS could decrease the efficiency of particle size reduction of BCA (S4-2 and S5-2) during homogenization. Furthermore, the particle sizes of BCA-NS/NCCS for S7-2 and S8-2 were significantly increased compared with those of S6-2. However, the particle size $D_{50}$ of BCA-NS with $0.5 \%$ SCS (S6-3) was $913.18 \pm 0.21 \mathrm{~nm}$ and increased to $2.183 \pm 0.74 \mu \mathrm{m}$ after storage. This indicated that only SCS was not able to effectively stabilize the drug nanocrystals, which was consistent with a previous report. ${ }^{19}$ The optimum BCA-NS/NCCS (S6-2) before and after storage exhibited the smallest mean particle size $(158 \pm 12.9 \mathrm{~nm})$ among all the formulations. Therefore, combination of NCC and SCS could play an important role in stabilizing BCA nanocrystals.

As shown in Figure 4, the zeta potential of BCA-NS/ NCCS (S1-2-S8-2) was -46.7 to $-66.9 \mathrm{mV}$. The results showed that with an increase in SCS concentration, the zeta potential value of BCA-NS/NCCS decreased. The zeta potential of the optimum BCA-NS/NCCS (S6-2) was $-60.7 \pm 1.4 \mathrm{mV}$, indicating a relatively low zeta potential of less than $-30 \mathrm{mV}$, which meant the good physical stability of nanosuspensions. ${ }^{46}$ These findings also demonstrated that the moderate concentration (S6-2) of NCCS could be beneficial for formation and stability of BCA-NS/NCCS, which might attribute to not only electrostatic repulsion effect but also the moderate viscosity. Therefore, the rheological behavior of different NCCSs was further characterized.

\section{Influence of different concentrations of dispersants on rheological evaluation of NCCS}

Rheological behavior (viscosity, modulus values, etc.) of the different NCCSs significantly affects the stability of NCCS and BCA-NS/NCCS during homogenization and storage. The excellent rheological behavior of NCCS can enhance the efficiency of particle size reduction and stability of BCANS/NCCS during homogenization. Therefore, the influence of NCCS concentrations on rheological characterization of NCCS was systemically investigated. In this study, the shear thinning behavior, storage modulus $\left(\mathrm{G}^{\prime}\right)$, and the loss modulus $\left(\mathrm{G}^{\prime \prime}\right)$ of different NCCS (S1-1-S8-1) and BCA-NS/ NCCS (S1-2-S8-2) suspensions were measured.

As illustrated in Figure 5A, the viscosity of suspension increased with the increase in the ratio of the SCS (S1-1-S5-1) and concentration of NCCS (S6-1-S8-1). Figure $5 \mathrm{~B}$ also shows that the viscosity of BCA-NS/NCCS also increased with the increase in the ratio of the SCS (S1-2-S5-2) and concentration of NCCS (S6-2-S8-2). In addition, Figure 5 also shows that the influence of shear stress on the viscosity of the NCCS. At a relative lower shear rate (less than 0.1 seconds $^{-1}$ ), the applied force might be less than the interaction forces of NCC and SCS. At relatively higher shear rates (more than 10 seconds $^{-1}$ ), the applied force became greater than the interaction forces of NCC and SCS. The viscosity of the NCCS decreased with respect to the applied shear rate. The viscoelastic property of the NCCS
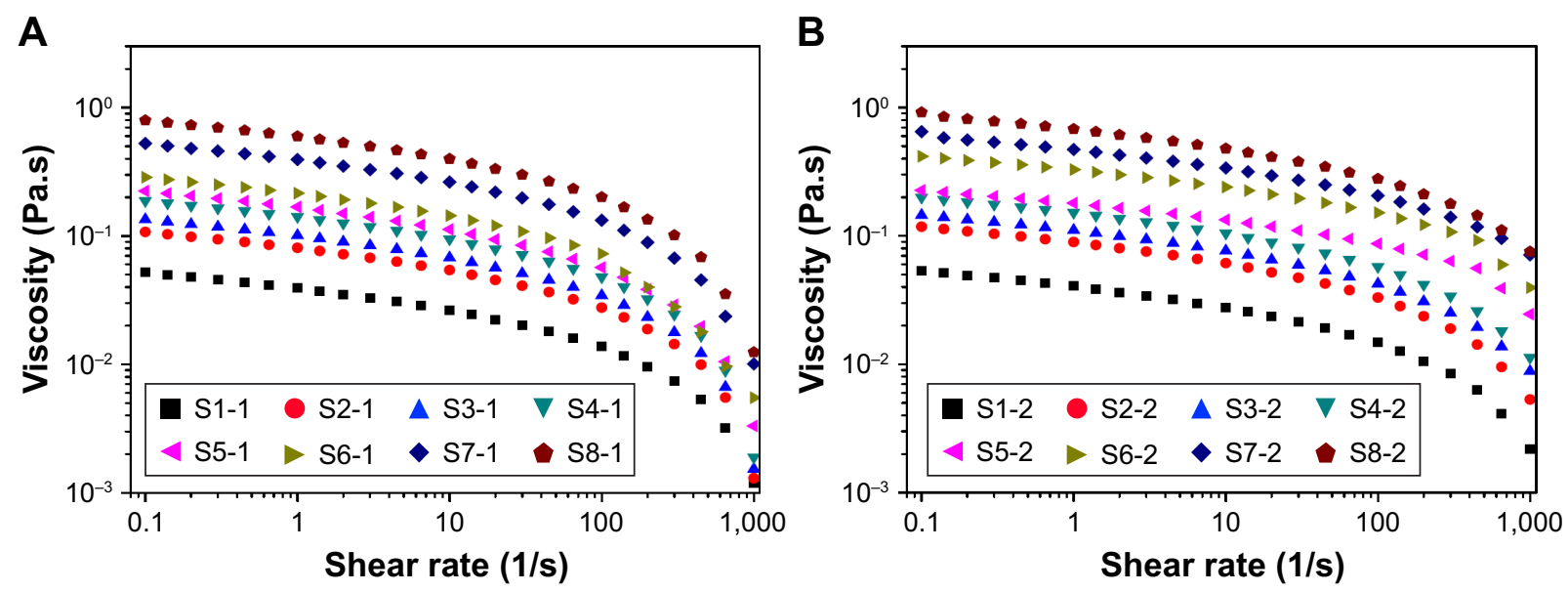

Figure 5 The shear thinning behavior of different NCCS (A) and BCA-NS/NCCS (B).

Abbreviations: BCA, baicalin; NCCS, nanocrystalline cellulose-sodium carboxymethyl starch; BCA-NS, BCA nanosuspension. 

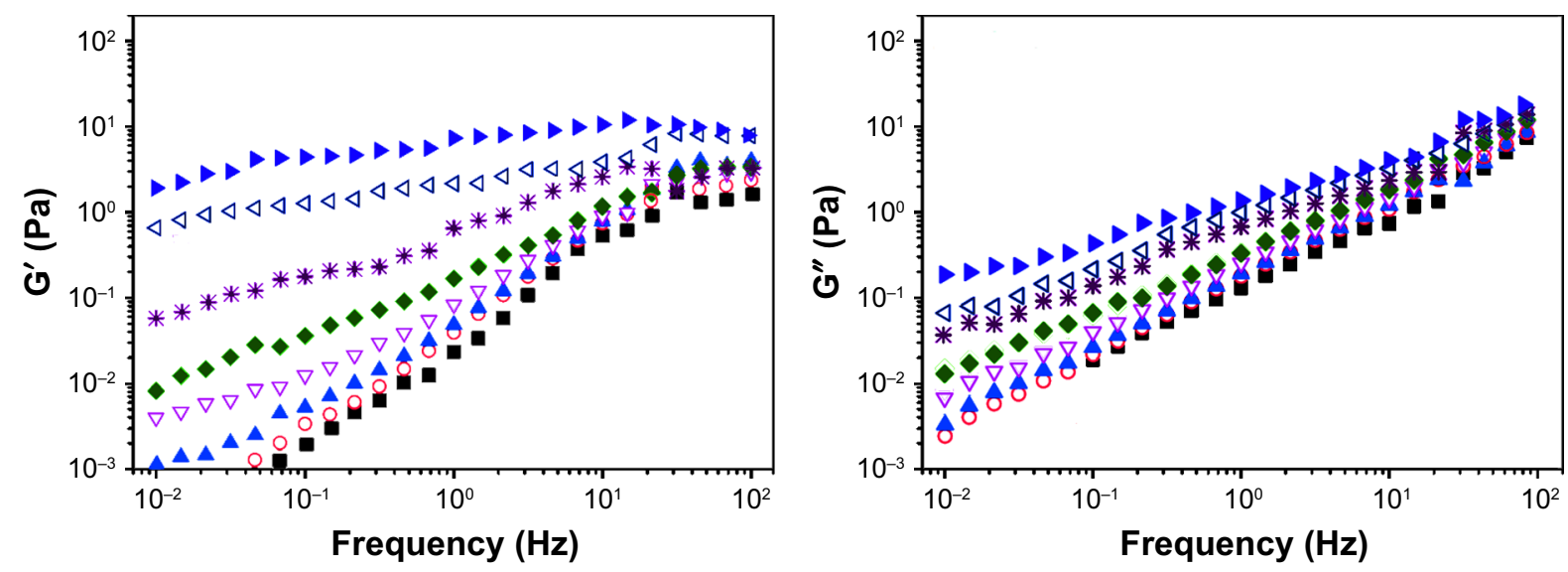

$\mathrm{S} 1-1 \quad \mathrm{O} 2-1 \quad \Delta \mathrm{S} 3-1 \quad \nabla \mathrm{S} 4-1$

S5-1

S6-1

$\triangleleft S 7-1$

S8-1

Figure 6 The viscoelastic property of the NCCS (SI-I-S8-I).

Abbreviation: NCCS, nanocrystalline cellulose-sodium carboxymethyl starch.

(S1-1-S8-1) was further characterized by the two dynamic moduli $\left(\mathrm{G}^{\prime}\right.$ and $\left.\mathrm{G}^{\prime \prime}\right)$, as illustrated in Figure 6. The results showed that $\mathrm{G}^{\prime}$ increasingly expanded as the concentrations of NCCS increased, which could be attributed to the increased particle-particle interactions of $\mathrm{NCC},{ }^{47}$ and the results also showed that at the lower NCCS concentration levels, $\mathrm{G}^{\prime \prime}$ was higher than $\mathrm{G}^{\prime}$, suggesting rather weak hydrodynamic interactions among the NCC particles. At the higher NCCS concentration levels (S7-1 and S8-1), G' showed higher values than $G^{\prime \prime}$ at the low frequency region, indicating the shear flow dominated by elastic deformation. Therefore, a high concentration of NCCS (1\% and 2\%) could exhibit the gel-like flow behavior, which could be responsible for the larger particle size of BCA-NS/NCCS (S7-2 and S8-2). ${ }^{48,49}$

\section{Influence of different concentrations of NCCS on stability and dissolution of BCA-NS/NCCS}

The dissolution profiles of the freshly prepared BCA-NS/ NCCS (S1-2-S8-2) are illustrated in Figure 7A. The results showed that the dissolution curve of BCA-NS/NCCS seemed to be different from that of the coarse BCA. The dissolution curve of BCA-NS/NCCS (S3-2 and S6-2) was significantly different from that of other formulations (S1-2, S2-2, S4-2,

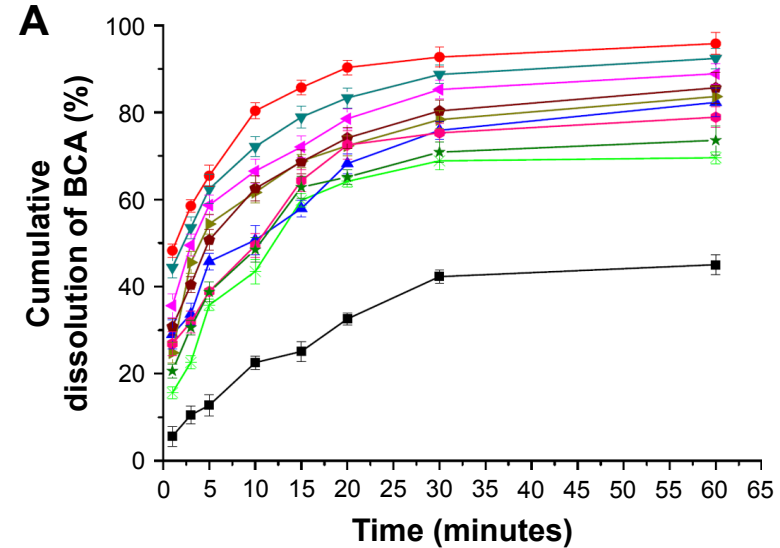

Time (minutes)

\begin{tabular}{|c|c|}
\hline$\rightarrow$ Coarse BCA & * BCA-NS/NCCS (S1-2) \\
\hline$\_$BCA-NS/NCCS (S2-2) & $\rightarrow$ BCA-NS/NCCS (S3-2) \\
\hline$\longleftarrow$ BCA-NS/NCCS (S4-2) & $\rightarrow$ BCA-NS/NCCS (S5-2) \\
\hline$\rightarrow$ BCA-NS/NCCS (S6-2) & $\rightarrow-B C A-N S / N C C S(S 7-2)$ \\
\hline$-\bullet$ BCA-NS/NCCS (S8-2) & \# BCA-NS/SCS (S6-3) \\
\hline
\end{tabular}

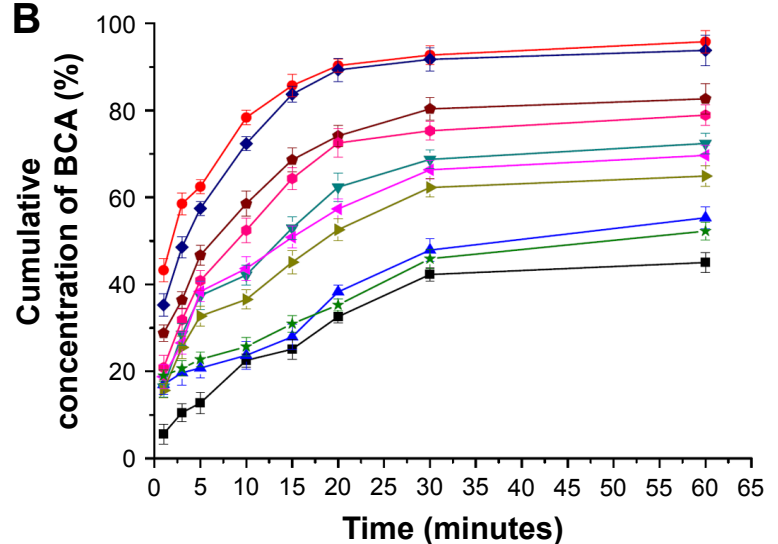

\begin{tabular}{|c|c|}
\hline$\rightarrow$ Coarse BCA & $\rightarrow$ BCA-NS/NCCS (S6-2) \\
\hline$\_$BCA-NP/NCCS (S2-2) & $\rightarrow$ BCA-NP/NCCS (S3-2) \\
\hline$\multimap$ BCA-NP/NCCS (S4-2) & $\rightarrow-B C A-N P / N C C S(S 5-2)$ \\
\hline$\leadsto-\mathrm{BCA}-\mathrm{NP} / \mathrm{NCCS}(\mathrm{S} 6-2)$ & $\rightarrow$ BCA-NP/NCCS (S7-2) \\
\hline$\rightarrow$ BCA-NP/NCCS (S8-2) & \# BCA-NP/SCS (S6-3) \\
\hline
\end{tabular}

Figure 7 In vitro dissolution profiles of BCA from different BCA-NS/NCCSs $(\mathbf{A})$ and BCA-NP/NCCSs (B).

Abbreviations: BCA, baicalin; BCA-NS, BCA nanosuspension; NCCS, nanocrystalline cellulose-sodium carboxymethyl starch; BCA-NP, BCA nanosuspension particle. 
S5-2, S6-3, S7-2, and S8-2; $f_{2}<50$ ). BCA-NS/NCCS (S3-2 and S6-2) exhibited the immediate release (more than 50\% at 5 minutes). These findings indicated that in comparison with individual NCC or SCS, the moderate concentration of NCCS could be very important for the stability and dissolution of BCA-NS/NCCS in the suspension state, which might be related to synergetic stabilization effect of NCC and SCS. However, the low concentration of NCCS could not effectively maintain the stability of BCA-NC, which might be responsible for poor dissolution of BCA-NS/NCCS (S1-2 and S2-2). The excessively high concentration of NCCS could significantly raise the viscosity of the BCA-NS/NCCS suspensions (as illustrated in Figures 5 and 6) and reduce the particles size reduction efficiency of BCA-NS/NCCS during homogenization, which could be the reason that the formulations (S4-2, S5-2, S7-2, and S8-2) exhibited the relative poor dissolution profiles.

The RDIs of BCA-NP/NCCS formulations with different ratios of NCCS are shown in Table 2. The results showed that RDI (1.12 \pm 0.05$)$ of BCA-NP/NCCS (S6-2) was less than 1.5, which indicated that BCA-NP/NCCS (S6-2) could recover back to the original BCA-NS/NCCS after rehydration. However, the RDIs of other formulations were more than 1.5 , which meant that the mean particle sizes of redispersed BCA-NS/NCCS could be increased, due to aggregation of BCA nanocrystals during spray drying. To further evaluate the redispersibility of BCA-NP/NCCS, in vitro dissolution of BCA-NP/NCCS formulations was investigated. The dissolution profiles of spray-dried BCA-NP/NCCS (S2-2-S8-2) are illustrated in Figure 7B. The results showed that the dissolution curve of spray-dried BCA-NP/NCCS (S6-2) seemed to be not significantly different from that of the freshly prepared BCA-NS/NCCS (S6-2; $f_{2}>50$ ); this was consistent with the RDI result of BCA-NP/NCCS (S6-2). The spray-dried BCA-NP/NCCS (S6-2) exhibited barely compromisingly fast dissolution. However, the dissolution curve of BCA-NS/ NCCS (S3-2, S4-2, or S5-2) was significantly different from that of the freshly prepared BCA-NS/NCCS $\left(\mathrm{S} 6-2 ; f_{2}<50\right)$ but was superior to that of BCA-NS/NCCS (S2-2). However, spray-dried BCA-NP/0.5\% SCS (S6-3) exhibited a very poor dissolution compared to BCA-NP/NCCS (S6-2; $f_{2}<50$ ). As evidenced in Table 2 and Figure 7, $0.5 \%$ concentration of NCCS (NCC:SCS=60:40) could enhance the redispersibility and dissolution of spray-dried BCA-NP/NCCS. However, $1 \%$ and $2 \%$ concentrations of NCCS could not effectively improve the dissolution of spray-dried BCA-NP/NCCS (S7-2 and S8-2). This compromising dissolution might be related to the larger size and higher viscosity of the redispersed BCA-NS/NCCS, as evidenced by the rheological results of NCCS (Table 2 and Figure 6).

It was concluded that, compared with the individual MCC or SCS, $0.5 \%$ concentration of NCCS (NCC:SCS=60:40) as a stabilizer could significantly improve dissolution performance of BCA-NP/NCCS, which might be attributed to (1) the smaller mean diameter of BCA-NS/NCCS during homogenization; (2) the appropriate viscosity, strong electrostatic repulsion, and/or steric barrier effect, which ensured the short-term suspension stability and prevented BCA-NP from aggregation during spray drying; and (3) the excellent swelling ability of SCS, which facilitated the fast disintegration and redispersion of BCA-NP after drying. ${ }^{50}$ Therefore, the optimum BCA-NP/NCCS (S6-2) was further characterized as follows.

\section{The morphology of NCCS, BCA-NS/ NCCS, and BCA-NP/NCCS}

The particle size of NCCS is shown in Figure 8A. NCCS seemed to be a colloidal solution. The mean particle size of NCCS was $147.12 \pm 0.14 \mathrm{~nm}$. TEM image of as-obtained NCCS is shown in Figure 8B. The NCCS exhibited the relatively homogeneously rod-like cellulose particles. Aggregation of NCC particles was not found, which could be attributed to presence of SCS. The SCS could effectively maintain the dispersing stability of NCC, as illustrated in Figure 8C.

The particle size of BCA-NS/NCCS is shown in Figure $8 \mathrm{D}$. BCA-NS/NCCS seemed to be suspensions. The mean particle size of BCA-NS/NCCS was $\sim 212.21 \pm 0.14 \mathrm{~nm}$. The TEM image of as-obtained NCCS is shown in Figure 8E. The BCA-NS/NCCS seemed to be near-sphere-shaped particles, and the needle-shaped NCC could be found on the surface of BCA nanocrystals. But the particle size of needle-shaped NCC (Figure 8E) adsorbed onto the surface of BCA nanocrystals seemed to be smaller than the original NCCS particle size (Figure 8B). It might be the reason that NCCS was again subjected to homogenization effect during

Table 2 RDI of BCA-NP/NCCS formulations with different ratios of NCCS

\begin{tabular}{l|l|l|l|l|l|l|l}
\hline & S2-2 & S3-2 & S4-2 & S5-2 & S6-2 & S7-2 \\
\hline RDI & $3.35 \pm 0.15$ & $2.34 \pm 0.08$ & $2.56 \pm 0.1 I$ & $2.82 \pm 0.12$ & $1.12 \pm 0.05$ & $1.61 \pm 0.06$ & $1.89 \pm 0.08$ \\
\hline
\end{tabular}

Abbreviations: BCA, baicalin; RDI, redispersibility index; BCA-NP, BCA nanosuspension particle; NCCS, nanocrystalline cellulose-sodium carboxymethyl starch. 

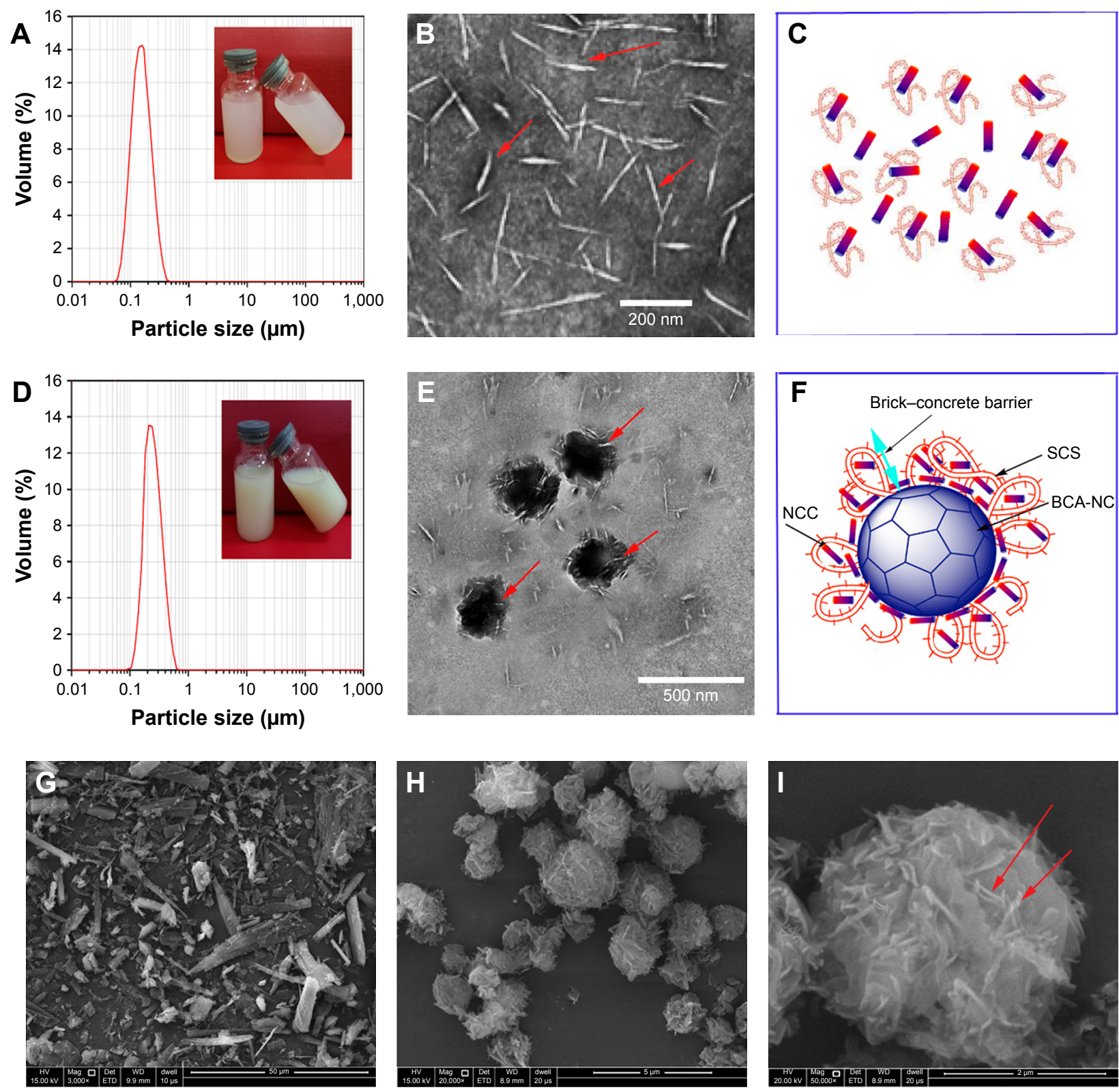

Figure 8 Particle size of NCCS (A), TEM morphology of NCCS (B), diagram of NCCS (C), particle size of BCA-NS/NCCS (D), TEM morphology of BCA-NS/NCCS (E), diagram of BCA-NS/NCCS (F), SEM of coarse BCA (G), SEM of BCA-NP/NCCS $(20,000 \times, \mathbf{H})$, and SEM of BCA-NP/NCCS $(50,000 \times, \mathbf{I})$.

Abbreviations: BCA, baicalin; NCCS, nanocrystalline cellulose-sodium carboxymethyl starch; TEM, transmitting electron microscopy; BCA-NS, BCA nanosuspension; SEM, scanning electron microscopy; BCA-NP, BCA nanosuspension particle.

production of BCA-NS/NCCS, which could further lead to the decrease in NCC particle size in the NCCS. As illustrated in Figure 8F, it could be speculated that this NCCS-based synergetic steric stabilizer could form the distinctive "brickconcrete" barrier shell around BCA-NC, and the presence of NCC might enhance the strength of steric barrier of polymer SCS. ${ }^{28}$ This brick-concrete barrier effect of NCCS could maintain the stability of BCA-NS/NCCS and prevent particles from aggregation, as evidenced by particle size comparison of BCA-NS/NCC (S1-2), BCA-NS/NCCS (S6-2), and BCA-NS/SCS (S6-3) in Figure 3. Therefore novel NCCS-based nanosuspensions could be named as Pickering nanosuspensions in this study, as referenced to the definition of Pickering emulsions. ${ }^{22,23}$

Figure $8 \mathrm{G}-\mathrm{I}$ shows the SEM images of coarse BCA and BCA-NP/NCCS. The results show that coarse BCA appears as a large blocky-shaped particle with particle sizes of 20-50 $\mu \mathrm{m}$ (Figure 8G). However, the BCA-NP/NCCS seemed to be sphere-shaped particles with particle sizes of $2-5 \mu \mathrm{m}$ (Figure $8 \mathrm{H}$ ). In addition, the presence of NCC on 
the surface of BCA-NP/NCCS could be found (Figure 8I). However, owing to their small particle size, BCA-NP/NCCS seemed to be cohesive, poorly flowing, and difficult to process. The flow ability of BCA-NP/NCCS could be further improved in the next work, in order to process into dried nanosuspension form.

\section{Characterization of solid state of NCCS and BCA-NP/NCCS DSC}

Figure 9 shows the thermal behavior of raw MCC, SCS, the physical mixture of MCC and SCS, NCCS, coarse BCA, the physical mixture of NCCS as well as BCA, and BCA-NP/ NCCS powders between $30^{\circ} \mathrm{C}$ and $260^{\circ} \mathrm{C}$. As can be seen, MCC showed wide endothermic peak in the investigated range (Figure 9A), which was attributed to its hierarchical organization (crystalline and amorphous fractions). ${ }^{43,44}$ SCS and the physical mixture of MCC and SCS all showed wide endothermic peak (Figure 9B and C). NCCS exhibited similar thermal behavior in comparison to that of physical mixture of MCC and SCS, but the enthalpy of NCCS was decreased (Figure 9D). Coarse BCA exhibited a sharp melting peak at $220^{\circ} \mathrm{C}$ (Figure 9E). The physical mixture of BCA and NCCS exhibited a characteristic melting peak of BCA at $220^{\circ} \mathrm{C}$ (Figure 9F). For the spray-dried BCA-NP/NCCS, the melting peak to $\mathrm{BCA}$ was shifted to $210^{\circ} \mathrm{C}-220^{\circ} \mathrm{C}$ (Figure $9 \mathrm{G}$ ),

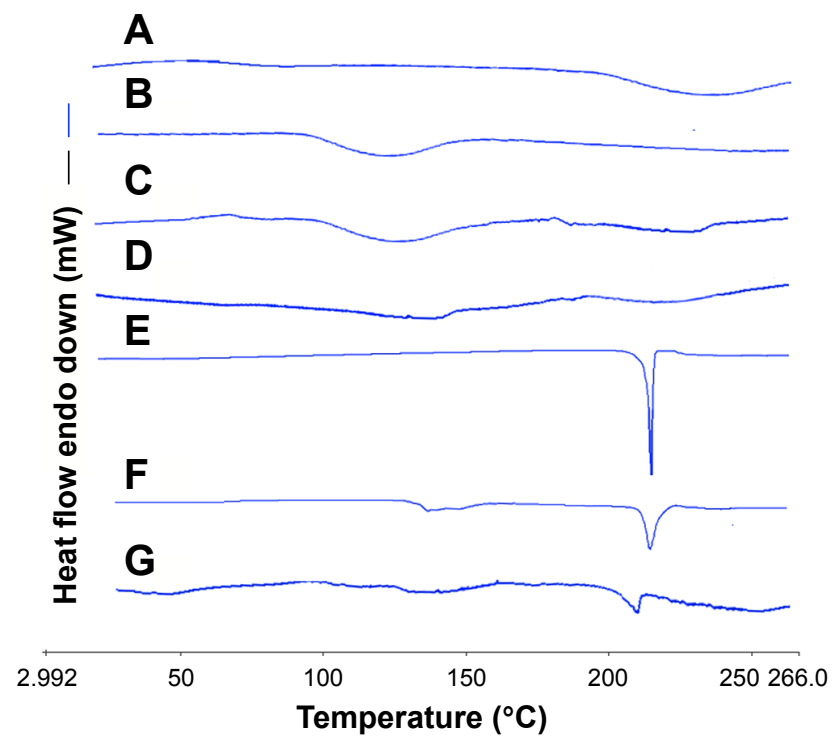

Figure 9 DSC thermograms of raw MCC (A), SCS (B), the physical mixture of MCC and SCS (C), NCCS (D), coarse BCA (E), the physical mixture of NCCS and $\mathrm{BCA}(\mathbf{F})$, and BCA-NP/NCCS $(\mathbf{G})$.

Abbreviations: DSC, differential scanning calorimetry; MCC, microcrystalline cellulose; SCS, sodium carboxymethyl starch; NCCS, nanocrystalline cellulose-sodium carboxymethyl starch; BCA, baicalin; BCA-NP, BCA nanosuspension particle. which indicated that the thermal behavior of BCA was not significantly changed compared with that of coarse BCA and the physical mixture of BCA as well as NCCS. This indicated that the applied physical treatment during the homogenization and presence of SCS could not alter crystal state of MCC or BCA. However, the enthalpy of NCCS and BCA-NP/NCCS was slightly decreased, possibly attributed to crystal-defect formation and/or particle size reduction..$^{51}$

\section{XRD}

Figure 10 displays the XRD diffractograms of raw $\mathrm{MCC}$, SCS, the physical mixture of MCC and SCS, NCCS, coarse BCA, the physical mixture of NCCS as well as BCA, and BCA-NP/NCCS powders. Raw MCC exhibited characteristic peaks at $2 \theta$ of $16.3^{\circ}, 22.6^{\circ}$, and $33.7^{\circ}$ (Figure 10A), which was a predominance of type I cellulose. ${ }^{52}$ SCS did not exhibit characteristic peaks (Figure 10B). The physical mixture of MCC and SCS exhibited the obvious characterization peak of MCC (Figure 10C). NCCS had similar characteristic crystalline peaks (Figure 10D) compared with raw MCC and physical mixture of MCC as well as SCS. There still existed the characteristic peak of NCC at the same $2 \theta$ position demonstrating that the crystal state of NCC was not changed during homogenization. The decrease in peak relative intensities was probably attributed to the size reduction of NCC in comparison with that of MCC.

Coarse BCA exhibited crystalline peaks at $2 \theta$ of $8.2^{\circ}$, $13.6^{\circ}, 15.4^{\circ}, 23.2^{\circ}, 27.5^{\circ}$, and $28.1^{\circ}$ (Figure 10E). The physical mixture of BCA and NCCS showed distinct crystalline peaks similar to coarse BCA (Figure 10F). BCA-NP/ NCCS showed distinct crystalline peaks similar to physical mixture of BCA and NCCS (Figure 10G). Peak positions of $\mathrm{BCA}$ remained unchanged compared to those of coarse $\mathrm{BCA}$, but with a lower peak intensity, which could be attributed to the aforementioned crystal-defect formation and/or particlesize reduction during homogenization. ${ }^{53}$

\section{Stability of BCA-NP/NCCS}

The stability of the spray-dried BCA-NP/NCCS was studied upon storage for 6 months. The dissolution behaviors of BCA-NP/NCCS stored at $4^{\circ} \mathrm{C}$ and $25^{\circ} \mathrm{C}$ were not obviously different compared with those of freshly prepared BCA-NP/ NCCS (Figure 11A). As illustrated in Figure 11B, it might be the reason that SCS with a high swelling capability was an effective dispersant to enhance the redispersion and fast dissolution of BCA-NP via a swelling-triggered erosion/ disintegration mechanism. ${ }^{45}$ The dissolution behavior of 

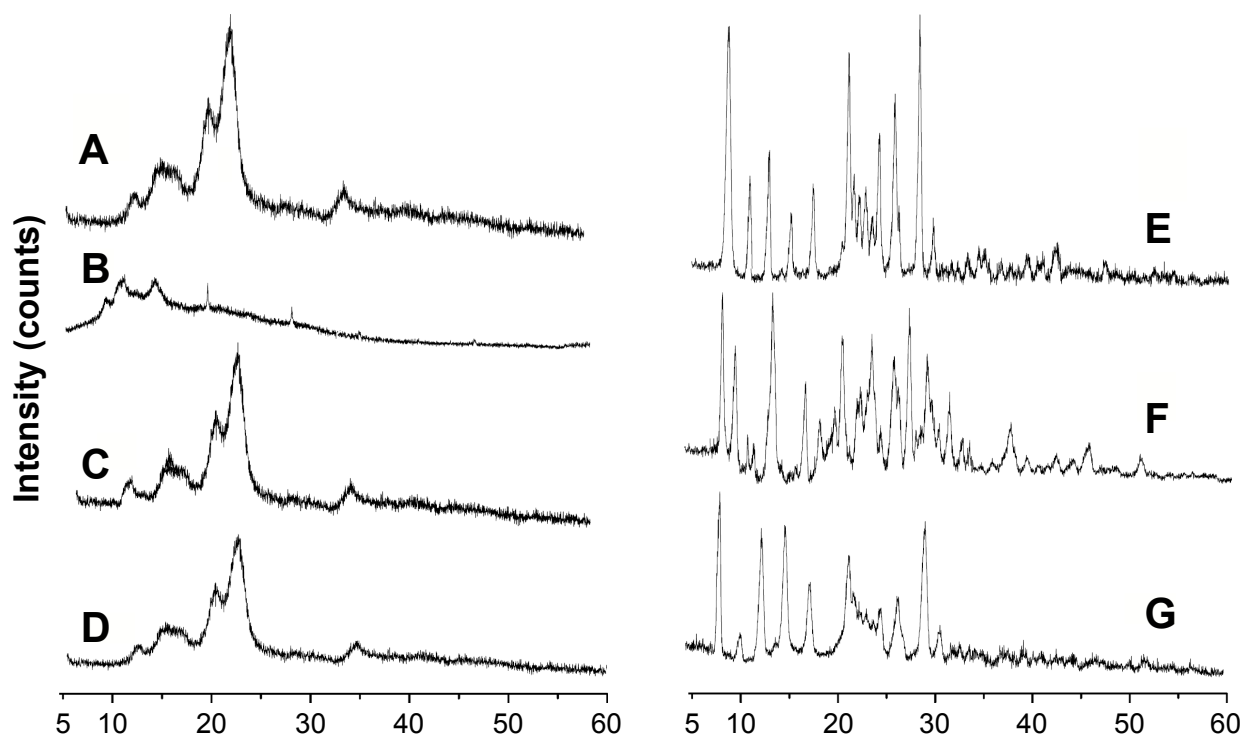

Figure 10 XRD of MCC raw MCC (A), SCS (B), the physical mixture of MCC and SCS (C), NCCS (D), coarse BCA (E), the physical mixture of NCCS and BCA (F), and BCA-NP/NCCS (G).

Abbreviations: XRD, X-ray diffraction; MCC, microcrystalline cellulose; SCS, sodium carboxymethyl starch; NCCS, nanocrystalline cellulose-sodium carboxymethyl starch; BCA, baicalin; BCA-NP, BCA nanosuspension particle.

BCA-NP/NCCS stored at $40^{\circ} \mathrm{C}$ was different compared with that of freshly prepared BCA-NP/NCCS $\left(f_{2}<50\right)$. However, BCA content of BCA-NP/NCCS was relatively stable at applied storage conditions. It might be the reason that NCCS adsorbing onto the surface of BCA nanocrystals could protect $\mathrm{BCA}$ from degradation during storage. ${ }^{54,55}$
The RDIs of BCA-NP/NCCS stored at $4{ }^{\circ} \mathrm{C}, 25^{\circ} \mathrm{C}$, and $40^{\circ} \mathrm{C}$ were $1.05 \pm 0.06,1.12 \pm 0.05$, and $1.65 \pm 0.08$. Therefore, BCANP/NCCS could form large aggregation owing to storage at a high temperature of $40^{\circ} \mathrm{C}$. This could be responsible for the compromising dissolution of BCA-NP/NCCS stored at $40^{\circ} \mathrm{C}$. The long-term stability of BCA-NP/NCCS and the

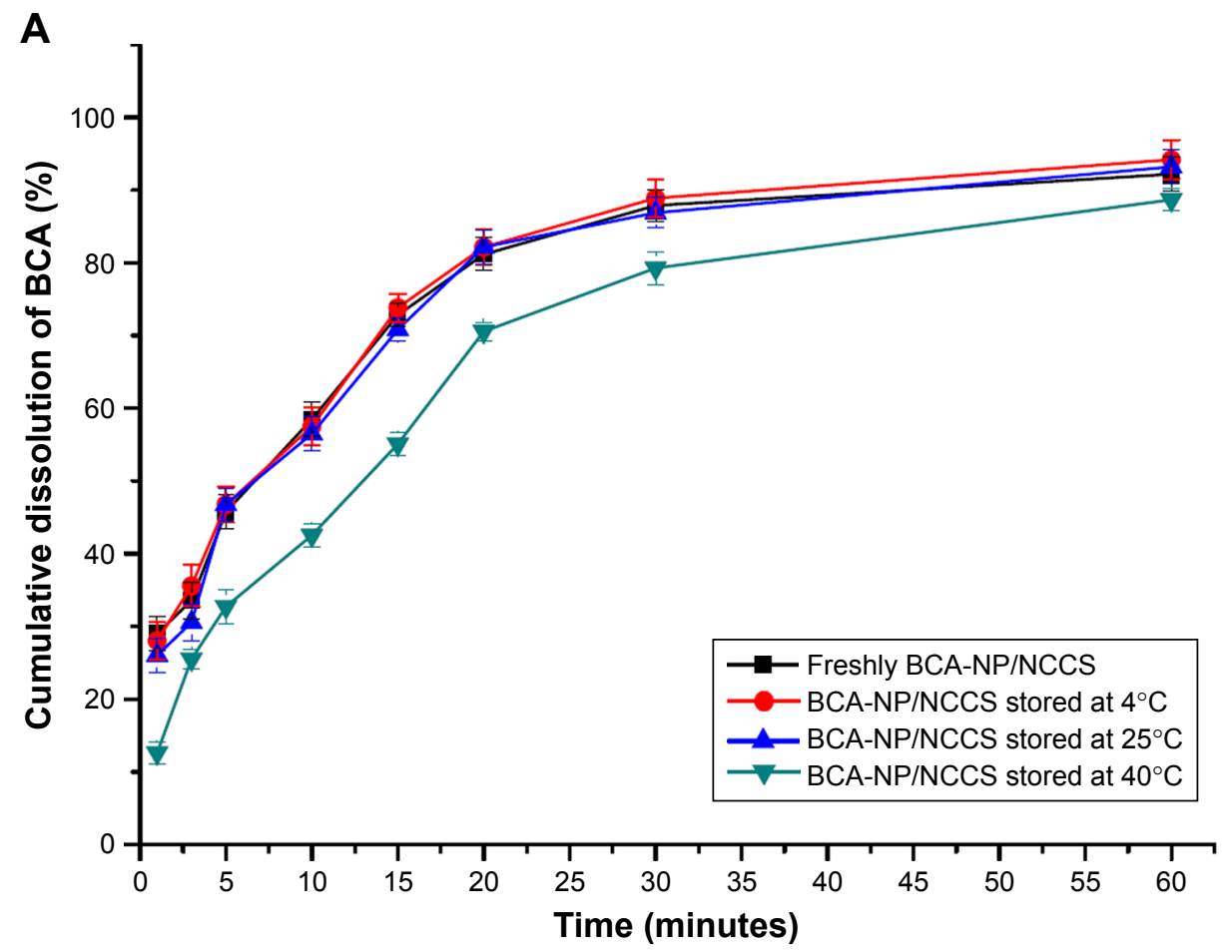

Figure I I (Continued) 
B

\begin{tabular}{|l} 
NCCS \\
SCS \\
Nanocrystals
\end{tabular}

Nanocrystals

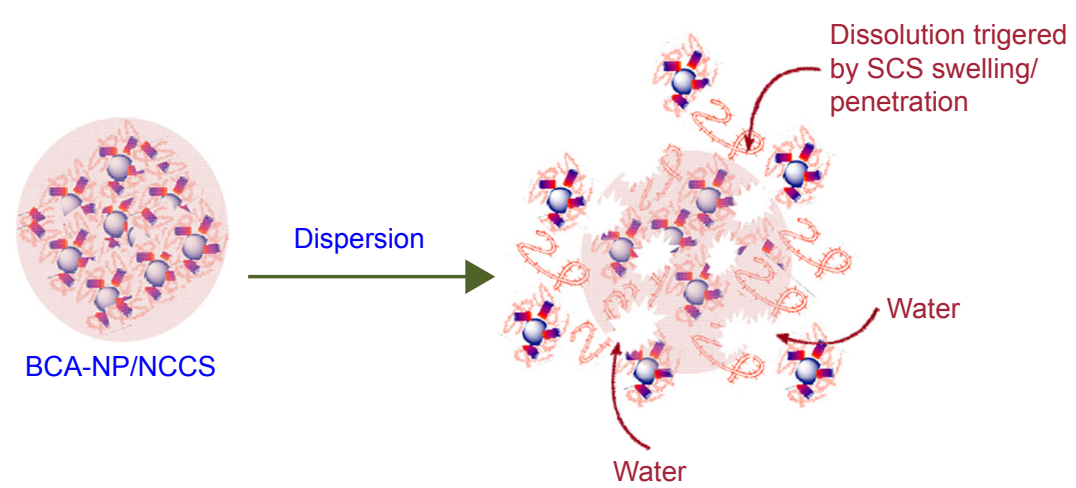

Figure II In vitro dissolution profiles of BCA from the spray-dried BCA-NP/NCCS after storage (A) and the schematic image on redispersion of BCA-NP/NCCS (B). Abbreviations: BCA, baicalin; BCA-NP, BCA nanosuspension particle; NCCS, nanocrystalline cellulose-sodium carboxymethyl starch; SCS, sodium carboxymethyl starch.

detailed mechanism would be further investigated in the follow-up study.

\section{The in vivo pharmacokinetic evaluation of BCA-NS/NCCS and BCA-NP/NCCS}

Pharmacokinetic studies of BCA-NP were investigated in rats in order to investigate whether the BCA-NP/NCCS could improve oral bioavailability of BCA in vivo compared with that of precursor BCA-NS/NCCS and crude BCA. The plasma concentration vs time curves of the BCA-NP, BCA-NS/NCCS, and the crude BCA are shown in Figure 12. After oral administration of these three different formulations, their individual mean plasma concentration-time curve profile showed a biphasic absorption phenomenon, which was attributed to the enterohepatic circulation of BCA. ${ }^{36}$

It could be seen that the plasma concentrations of BCANP/NCCS were significantly higher within 5 hours after

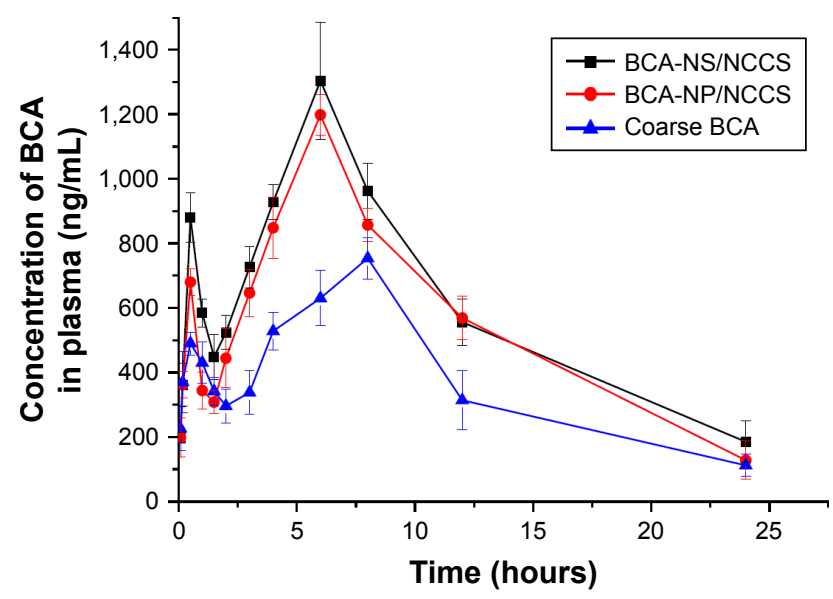

Figure 12 Mean plasma concentration-time profiles in rats after oral administration of coarse BCA, BCA-NP/NCCS, and BCA-NS/NCCS at $100 \mathrm{mg} / \mathrm{kg}(\mathrm{n}=6)$. Abbreviations: BCA, baicalin; BCA-NP, BCA nanosuspension particle; NCCS, nanocrystalline cellulose-sodium carboxymethyl starch; BCA-NS, BCA nanosuspension. administration compared with those of crude $\mathrm{BCA}(P<0.05)$ but not significantly different from those of BCA-NS/ NCCS $(P>0.05)$. The main pharmacokinetic parameters of the BCA-NP, BCA-NS/NCCS, and the crude BCA are listed in Table 3 . The mean peak concentration $\left(C_{\max }\right)$ of the BCA-NS/NCCS $(1,198.46 \pm 113.81 \mu \mathrm{g} / \mathrm{L})$ and BCANP/NCCS $(1,304.81 \pm 123.79 \mu \mathrm{g} / \mathrm{L})$ was significantly increased $(P<0.05)$ compared with that of the crude BCA $(630.83 \pm 86.48 \mu \mathrm{g} / \mathrm{L})$. As listed in Table 3, the $\mathrm{AUC}_{(0-\infty)}$ of the BCA-NP/NCCS $(8,773.38 \pm 718.18 \mu \mathrm{g} / \mathrm{L} \cdot \mathrm{h})$ was 2.01 times $(P<0.05)$ as high as that of the coarse BCA $(4,354.61 \pm 451.28 \mu \mathrm{g} / \mathrm{L} \cdot \mathrm{h})$. However, the main pharmacokinetic parameters $\left(C_{\max }\right.$ and $\left.\mathrm{AUC}_{(0-\infty)}\right)$ of BCA-NP/NCCS were not significantly different from those of BCA-NS/NCCS $(P>0.05)$, which is also consistent with that in the dissolution studies described earlier. BCA-NP/NCCS could easily recover back to the BCA-NS/NCCS in the gastrointestinal tract after oral administration. It could be reason that the reduced particle size of BCA nanocrystals along with an increase in surface area significantly increased the dissolution rate and oral bioavailability of BCA. Therefore, the reduced particle size could play an important role in dissolution and gastrointestinal absorption..$^{56,57}$

\section{Conclusion}

The novel nanosuspensions using co-processed NCCS as a steric stabilizer were successfully prepared by homogenization technology, with the goal of enhancing dissolution of poorly soluble drug. NCCS could be beneficial for formation and stability of BCA-NS/NCCS, which might attribute to the brick-concrete-based steric barrier composed of NCC particles and SCS polymer. Furthermore, the optimum NCCS could completely prevent aggregation of BCA nanocrystals during spray drying and enhance the redispersibility as well as 
Table 3 Main pharmacokinetic parameters of coarse BCA, BCA-NS/NCCS, and BCA-NP/NCCS after oral administration in rats $(\mathrm{n}=6, \bar{x} \pm s)$

\begin{tabular}{|c|c|c|c|c|}
\hline & Unit & BCA & BCA-NS/NCCS & BCA-NP/NCCS \\
\hline $\mathrm{MRT}_{(0-\infty)}$ & Hours & $7.92 \pm 0.24$ & $7.58 \pm 0.5 \mathrm{I}$ & $6.87 \pm 1.45^{*}$ \\
\hline$T_{\max }$ & Hours & $7.62 \pm 0.89$ & $6.85 \pm 1.77$ & $7.24 \pm 1.05^{*}$ \\
\hline$C_{\max }$ & $\mathrm{ng} \cdot \mathrm{L}^{-1}$ & $630.83 \pm 86.48$ & $\mathrm{I},|98.46 \pm| \mid 3.8 \mathrm{I}^{*}$ & $\mathrm{I}, 304.8 \mathrm{I} \pm \mathrm{I} 23.79 *$ \\
\hline$t_{1 / 2}$ & Hours & $5.26 \pm 1.29$ & $3.42 \pm 0.5 \mathrm{I}$ & $4.57 \pm 0.87$ \\
\hline $\mathrm{AUC}_{(0-24)}$ & $\mu \mathrm{g} /(\mathrm{L} \cdot \mathrm{h})$ & $4,354.6 \mathrm{I} \pm 45 \mathrm{I} .28$ & $8,036.42 \pm 510.16^{* *}$ & $8,773.38 \pm 7 \mid 8.18^{* *}$ \\
\hline
\end{tabular}

Notes: $* P<0.05$ and $* * P<0.01$ vs control group. $C_{\max }$, mean peak concentration.

Abbreviations: BCA, baicalin; BCA-NS, BCA nanosuspension; NCCS, nanocrystalline cellulose-sodium carboxymethyl starch; BCA-NP, BCA nanosuspension particle; $M R T_{(0-\infty)}$, mean residence time; $T_{\text {max }}$, peak time; $t_{1 / 2}$, half-life time; $A \cup C_{(0-24)}$, area under the curve.

dissolution of spray-dried BCA-NP/NCCS. The crystal state of NCC and BCA presented in BCA-NP/NCCS remained unchanged during the homogenization. The BCA-NP/ NCCS exhibited excellent redispersibility and significantly a fast dissolution rate of BCA. This study demonstrated that novel surfactant-free nanosuspensions could be prepared by co-processed NCCS and also provided a feasible strategy to improve dissolution of poorly soluble drug.

\section{Acknowledgment}

The authors would like to acknowledge the financial support from the Scientific Research Foundation for the National Natural Science Foundation of China (81560656 and 81760715), the Young Jinggang Scholar Award Program and Fund of Distinguished Young Scientists of Jiangxi Province (20162BCB23033), and the Natural Science Fund of Jiangxi Province (20161BAB205221).

\section{Disclosure}

The authors report no conflicts of interest in this work.

\section{References}

1. Lu Y, Li Y, Wu W. Injected nanocrystals for targeted drug delivery. Acta Pharm Sin B. 2016;6(2):106-113.

2. Raghava Srivalli KM, Mishra B. Drug nanocrystals: A way toward scale-up. Saudi Pharm J. 2016;24(4):386-404.

3. Huang $\mathrm{S}$, Zhang Q, Li H, et al. Increased bioavailability of efonidipine hydrochloride nanosuspensions by the wet-milling method. Eur $J$ Pharm Biopharm. 2018;130:108-114.

4. Wang Y, Zheng Y, Zhang L, Wang Q, Zhang D. Stability of nanosuspensions in drug delivery. J Control Release. 2013;172(3):1126-1141.

5. Yang H, Kim H, Jung S, et al. Pharmaceutical strategies for stabilizing drug nanocrystals. Curr Pharm Des. 2018;24(21):2362-2374.

6. Geng T, Banerjee P, Lu Z, Zoghbi A, Li T, Wang B. Comparative study on stabilizing ability of food protein, non-ionic surfactant and anionic surfactant on BCS type II drug carvedilol loaded nanosuspension: Physicochemical and pharmacokinetic investigation. Eur J Pharm Sci. 2017;109:200-208.

7. Knieke C, Rawtani A, Davé RN. Concentrated fenofibrate nanoparticle suspensions from melt emulsification for enhanced drug dissolution. Chem Eng Technol. 2014;37(1):157-167.

8. Toziopoulou F, Malamatari M, Nikolakakis I, Kachrimanis K. Production of aprepitant nanocrystals by wet media milling and subsequent solidification. Int J Pharm. 2017;533(2):324-334.
9. Malamatari M, Somavarapu S, Taylor KM, Buckton G. Solidification of nanosuspensions for the production of solid oral dosage forms and inhalable dry powders. Expert Opin Drug Deliv. 2016;13(3):435-450.

10. Zhang Y, Feng J, McManus SA, et al. Design and solidification of fastreleasing clofazimine nanoparticles for treatment of cryptosporidiosis. Mol Pharm. 2017;14(10):3480-3488.

11. Yue PF, Li G, Dan JX, et al. Study on formability of solid nanosuspensions during solidification: II novel roles of freezing stress and cryoprotectant property. Int J Pharm. 2014;475(1-2):35-48.

12. Alaei S, Ghasemian E, Vatanara A. Spray drying of cefixime nanosuspension to form stabilized and fast dissolving powder. Powder Technol. 2016;288:241-248.

13. van Eerdenbrugh B, Froyen L, van Humbeeck J, Martens JA, Augustijns P, van den Mooter G. Alternative matrix formers for nanosuspension solidification: Dissolution performance and X-ray microanalysis as an evaluation tool for powder dispersion. Eur J Pharm Sci. 2008;35(4):344-353.

14. Bilgili E, Afolabi A. A combined microhydrodynamics-polymer adsorption analysis for elucidation of the roles of stabilizers in wet stirred media milling. Int J Pharm. 2012;439(1-2):193-206.

15. Liversidge GG, Cundy KC. Particle size reduction for improvement of oral bioavailability of hydrophobic drugs: I. Absolute oral bioavailability of nanocrystalline danazol in beagle dogs. Int J Pharm. 1995; 125(1):91-97.

16. Suzuki M, Machida M, Adachi K, et al. Histopathological study of the effects of a single intratracheal instillation of surface active agents on lung in rats. J Toxicol Sci. 2000;25(1):49-55.

17. Lebhardt T, Roesler S, Uusitalo HP, Kissel T. Surfactant-free redispersible nanoparticles in fast-dissolving composite microcarriers for dry-powder inhalation. Eur J Pharm Biopharm. 2011;78(1):90-96.

18. Ma Y, Yang Y, Xie J, Xu J, Yue P, Yang M. Novel nanocrystal-based solid dispersion with high drug loading, enhanced dissolution, and bioavailability of andrographolide. Int J Nanomedicine. 2018;13:3763-3779.

19. Azad M, Afolabi A, Bhakay A, Leonardi J, Davé R, Bilgili E. Enhanced physical stabilization of fenofibrate nanosuspensions via wet co-milling with a superdisintegrant and an adsorbing polymer. Eur J Pharm Biopharm. 2015;94:372-385.

20. Seabra AB, Bernardes JS, Fávaro WJ, Paula AJ, Durán N. Cellulose nanocrystals as carriers in medicine and their toxicities: A review. Carbohydr Polym. 2018;181:514-527.

21. Sunasee R, Hemraz UD, Ckless K. Cellulose nanocrystals: a versatile nanoplatform for emerging biomedical applications. Expert Opin Drug Deliv. 2016;13(9):1243-1256.

22. Zoppe JO, Venditti RA, Rojas OJ. Pickering emulsions stabilized by cellulose nanocrystals grafted with thermo-responsive polymer brushes. J Colloid Interface Sci. 2012;369(1):202-209.

23. Kasiri N, Fathi M. Production of cellulose nanocrystals from pistachio shells and their application for stabilizing Pickering emulsions. Int $J$ Biol Macromol. 2018;106:1023-1031.

24. Yang X, Bakaic E, Hoare T, Cranston ED. Injectable polysaccharide hydrogels reinforced with cellulose nanocrystals: morphology, rheology, degradation, and cytotoxicity. Biomacromolecules. 2013;14(12): $4447-4455$. 
25. Lin N, Dufresne A. Supramolecular hydrogels from in situ host-guest inclusion between chemically modified cellulose nanocrystals and cyclodextrin. Biomacromolecules. 2013;14(3):871-880.

26. Bandera D, Sapkota J, Josset S, et al. Influence of mechanical treatments on the properties of cellulose nanofibers isolated from microcrystalline cellulose. Reactive and Functional Polymers. 2014;85:134-141.

27. Dan J, Ma Y, Yue P, et al. Microcrystalline cellulose-carboxymethyl cellulose sodium as an effective dispersant for drug nanocrystals: A case study. Carbohydr Polym. 2016;136:499-506.

28. Hu Z, Marway HS, Kasem H, Pelton R, Cranston ED, Emily D. Dried and Redispersible Cellulose Nanocrystal Pickering Emulsions. ACS Macro Lett. 2016;5(2):185-189.

29. Omidian H, Park K. Swelling agents and devices in oral drug delivery. J Drug Deliv Sci Technol. 2008;18(2):83-93.

30. Bilgili E, Li M, Afolabi A. Is the combination of cellulosic polymers and anionic surfactants a good strategy for ensuring physical stability of BCS Class II drug nanosuspensions? Pharm Dev Technol. 2016; 21(4):499-510.

31. Azad M, Arteaga C, Abdelmalek B, Davé R, Bilgili E. Spray drying of drug-swellable dispersant suspensions for preparation of fast-dissolving, high drug-loaded, surfactant-free nanocomposites. Drug Dev Ind Pharm. 2015;41(10):1617-1631.

32. Zhang Y, Zhang K, Guo T, Li Y, Zhu C, Feng N. Transdermal baicalin delivery using diethylene glycol monoethyl ether-mediated cubic phase gel. Int J Pharm. 2015;479(1):219-226.

33. Mir-Palomo S, Nácher A, Díez-Sales O, et al. Inhibition of skin inflammation by baicalin ultradeformable vesicles. Int J Pharm. 2016; 511(1):23-29.

34. Dinda B, Dinda S, Dassharma S, Banik R, Chakraborty A, Dinda M. Therapeutic potentials of baicalin and its aglycone, baicalein against inflammatory disorders. Eur J Med Chem. 2017;131:68-80.

35. Wei Y, Guo J, Zheng X, et al. Preparation, pharmacokinetics and biodistribution of baicalin-loaded liposomes. Int J Nanomedicine. 2014; 9:3623-3630.

36. Yue PF, Li Y, Wan J, et al. Process optimization and evaluation of novel baicalin solid nanocrystals. Int J Nanomedicine. 2013;8:2961-2973.

37. Liu W, Tian $\mathrm{R}, \mathrm{Hu} \mathrm{W}$, et al. Preparation and evaluation of selfmicroemulsifying drug delivery system of baicalein. Fitoterapia. 2012; 83(8):1532-1539.

38. Zhao L, Wei Y, Huang Y, He B, Zhou Y, Fu J. Nanoemulsion improves the oral bioavailability of baicalin in rats: in vitro and in vivo evaluation. Int J Nanomedicine. 2013;8:3769-3779.

39. Hao J, Wang F, Wang X, et al. Development and optimization of baicalinloaded solid lipid nanoparticles prepared by coacervation method using central composite design. Eur J Pharm Sci. 2012;47(2):497-505.

40. Chen H, Gao Y, Wu J, et al. Exploring therapeutic potentials of baicalin and its aglycone baicalein for hematological malignancies. Cancer Lett. 2014;354(1):5-11.

41. Liu Y, Ma Y, Xu J, et al. Apolipoproteins adsorption and brain-targeting evaluation of baicalin nanocrystals modified by combination of Tween 80 and TPGS. Colloids Surf B Biointerfaces. 2017;160:619-627.
42. Habibi Y, Lucia LA, Rojas OJ. Cellulose nanocrystals: chemistry, self-assembly, and applications. Chem Rev. 2010;110(6):3479-3500.

43. George J, Sabapathi SN. Cellulose nanocrystals: synthesis, functional properties, and applications. Nanotechnol Sci Appl. 2015;8:45-54.

44. Buffiere J, Balogh-Michels Z, Borrega M, Geiger T, Zimmermann T, Sixta H. The chemical-free production of nanocelluloses from microcrystalline cellulose and their use as Pickering emulsion stabilizer. Carbohydr Polym. 2017;178:48-56.

45. Bhakay A, Azad M, Vizzotti E, Dave RN, Bilgili E. Enhanced recovery and dissolution of griseofulvin nanoparticles from surfactant-free nanocomposite microparticles incorporating wet-milled swellable dispersants. Drug Dev Ind Pharm. 2014;40(11):1509-1522.

46. Li Q, Chen F, Liu Y, et al. A novel albumin wrapped nanosuspension of meloxicam to improve inflammation-targeting effects. Int J Nanomedicine. 2018;13:4711-4725.

47. Boluk Y, Zhao L, Incani V. Dispersions of nanocrystalline cellulose in aqueous polymer solutions: structure formation of colloidal rods. Langmuir. 2012;28(14):6114-6123.

48. Shafiei-Sabet S, Hamad WY, Hatzikiriakos SG. Rheology of nanocrystalline cellulose aqueous suspensions. Langmuir. 2012;28(49): 17124-17133.

49. Chen Y, Xu C, Huang J, Wu D, Lv Q. Rheological properties of nanocrystalline cellulose suspensions. Carbohydr Polym. 2017;157: 303-310.

50. Ma Y, Yang Y, Xie J, Xu J, Yue P, Yang M. Novel nanocrystal-based solid dispersion with high drug loading, enhanced dissolution, and bioavailability of andrographolide. Int J Nanomedicine. 2018;13: 3763-3779.

51. Kayaert $P$, van den Mooter $G$. Is the amorphous fraction of a dried nanosuspension caused by milling or by drying? A case study with Naproxen and Cinnarizine. Eur J Pharm Biopharm. 2012;81(3):650-656.

52. Silvério HA, Flauzino Neto WP, Dantas NO, Pasquini D. Extractionand characterization of cellulose nanocrystals from agro-industrial residue-Soy hulls. Industrial Crops and Products. 2013;42:480-488.

53. Ren X, Qi J, Wu W, Yin Z, Li T, Lu Y. Development of carrier-free nanocrystals of poorly water-soluble drugs by exploring metastable zone of nucleation. Acta Pharm Sin B. 2018.

54. Gao L, Liu G, Wang X, Liu F, Xu Y, Ma J. Preparation of a chemically stable quercetin formulation using nanosuspension technology. Int $J$ Pharm. 2011;404(1-2):231-237.

55. Möschwitzer J, Achleitner G, Pomper H, Müller RH. Development of an intravenously injectable chemically stable aqueous omeprazole formulation using nanosuspension technology. Eur J Pharm Biopharm. 2004;58(3):615-619.

56. Chen C, Wang L, Cao F, et al. Formulation of 20 (S)-protopanaxadiol nanocrystals to improve oral bioavailability and brain delivery. Int $J$ Pharm. 2016;497(1-2):239-247.

57. Lu Y, Qi J, Dong X, Zhao W, Wu W. The in vivo fate of nanocrystals. Drug Discov Today. 2017;22(4):744-750.

International Journal of Nanomedicine

\section{Publish your work in this journal}

The International Journal of Nanomedicine is an international, peerreviewed journal focusing on the application of nanotechnology in diagnostics, therapeutics, and drug delivery systems throughout the biomedical field. This journal is indexed on PubMed Central, MedLine, CAS, SciSearch $\AA$, Current Contents ${ }^{\circledR} /$ Clinical Medicine,

Journal Citation Reports/Science Edition, EMBase, Scopus and the Elsevier Bibliographic databases. The manuscript management system is completely online and includes a very quick and fair peer-review system, which is all easy to use. Visit http://www.dovepress.com/ testimonials.php to read real quotes from published authors. 